\title{
Unified Stochastic Geometry Model for MIMO Cellular Networks with Retransmissions
}

\author{
Laila Hesham Afify, Hesham ElSawy, Tareq Y. Al-Naffouri, and Mohamed-Slim Alouini \\ King Abdullah University of Science and Technology (KAUST) \\ Email: \{laila.afify, hesham.elsawy, tareq.alnaffouri, slim.alouini\}@kaust.edu.sa
}

\begin{abstract}
This paper presents a unified mathematical paradigm, based on stochastic geometry, for downlink cellular networks with multiple-input-multiple-output (MIMO) base stations (BSs). The developed paradigm accounts for signal retransmission upon decoding errors, in which the temporal correlation among the signal-to-interference-plus-noise-ratio (SINR) of the original and retransmitted signals is captured. In addition to modeling the effect of retransmission on the network performance, the developed mathematical model presents twofold analysis unification for MIMO cellular networks literature. First, it integrates the tangible decoding error probability and the abstracted (i.e., modulation scheme and receiver type agnostic) outage probability analysis, which are largely disjoint in the literature. Second, it unifies the analysis for different MIMO configurations. The unified MIMO analysis is achieved by abstracting unnecessary information conveyed within the interfering signals by Gaussian signaling approximation along with an equivalent SISO representation for the per-data stream SINR in MIMO cellular networks. We show that the proposed unification simplifies the analysis without sacrificing the model accuracy. To this end, we discuss the diversity-multiplexing tradeoff imposed by different MIMO schemes and shed light on the diversity loss due to the temporal correlation among the SINRs of the original and retransmitted signals. Finally, several design insights are highlighted.
\end{abstract}

Keywords-MIMO cellular networks, error probability, outage probability, ergodic rate, stochastic geometry, network design.

\section{INTRODUCTION}

Multiple-input-multiple-output (MIMO) transmission offers diverse options for antenna configurations that can lead to different diversity and multiplexing tradeoffs, which can be exploited to improve several aspects in wireless networks performance. For instance, link capacity gains are harvested by multiplexing several data streams into the same channel via MIMO spatial multiplexing. Enhanced link reliability is obtained by transmit and/or receive diversity. The network capacity is improved by accommodating more users equipment (UEs) per channel via multi-user MIMO techniques. Last but not least, enhanced interference management is achieved via beamforming or interference alignment schemes that suppress dominant interference sources at the receivers side, which improves the signal-to-interference-plus-noise ratio (SINR).

Motivated by its potential gains, MIMO is considered an essential ingredient in modern cellular networks and 3GPP standards to cope with the ever-growing capacity demands. However, the MIMO operation is understood and its associated gains are quantified for elementary network settings [1][3], which do not directly generalize to cellular networks. The

This work was funded by a CRG3 grant ORS\#221 from the Office of Competitive Research (OCRF) at King Abduallah University of Science and Technology (KAUST). operation of large-scale cellular network is highly affected by inter-cell interference, which emerges from spatial frequency reuse. Therefore, to characterize MIMO operation and quantify its potential gains in cellular network, the impact of perbase station (BS) precoding on the aggregate interference as well as the effect of the aggregate interference on the received SINR after MIMO post-processing should be characterized. Exploiting recent advances in stochastic geometry analysis, several mathematical frameworks are developed to study MIMO operation in cellular networks [4]-[20]. Stochastic geometry does not only provide systematic and tractable framework to model MIMO operation in interference environments, it also captures the behavior of realistic cellular networks as reported in [21]-[25]. The authors in [4] study the SINR coverage probability of orthogonal space-time block codes (OSTBC). Studies for the outage probability and ergodic rate for space-division-multiple-access (SDMA) MIMO, also known as multi-user MIMO (MU-MIMO), are available in [14], [15] for single-tier cellular networks and in [20] for multi-tier cellular networks. Coverage probability improvement via maximum-ratio-combining (MRC) with spatial interference correlation is quantified in [5]. The potential gains of beamforming and interference alignment in terms of SINR coverage and network throughput are quantified in [6]-[8]. Coverage probability and rate for MRC and optimum combining in uplink MIMO cellular networks are studied in [19]. Network MIMO via BS cooperation performance in terms of outage probability and ergodic rate are studied in [9]-[13]. Average symbol error probability (ASEP) and average pairwise error probability (APEP) for several MIMO configurations are studied in [16], [17]. Asymptotic analysis for minimum mean square error MIMO receivers is conducted in [18]. A general framework that can compare outage probability and rate for different MIMO schemes in advanced cellular network models is developed in [25].

Despite that the mathematical models presented in [4]-[18] are all based on stochastic geometry, there are significant differences in terms of the analysis steps as well as the level of details provided by each model. The majority of the models focus on the outage probability and ergodic capacity for simplicity [4]-[15], [19], [20], [25]. While both outage probability and ergodic rate are fundamental key performance indicators (KPIs) in wireless communication, they convey no information about the underlying modulation scheme, constellation size, or receiver type. Considering more tangible KPIs, such as decoding error probability and average throughput ${ }^{1}$, requires alternative and more involved analysis as shown in [16], [17]. The error probability analysis

\footnotetext{
${ }^{1}$ Throughput is defined as the number of successfully transmitted bits per channel use.
} 
is involved due to the necessity to model the aggregate interference signal at the complex baseband level and statistically account for the transmitted symbols from each interferer [26], [27]. Furthermore, the analysis in each of the mathematical models presented in [4]-[17], [19], [20] is highly dependent on the considered MIMO configuration. Having mathematical models that are significantly different in the analysis steps from one KPI to another and from one antenna configuration to another makes it challenging to conduct comprehensive studies that include different antenna configurations and compare their performances in terms of different KPIs. Furthermore, the effect of retransmission upon decoding errors on the network performance is only studied for cooperating single-antenna BSs [12], which is different from the case when both BSs and UEs are equipped with multiple antennas and precoding/combining is applied.

This paper presents a unified mathematical paradigm, based on stochastic geometry, to study the average error probability, outage probability, and ergodic rate for cellular networks with different MIMO settings ${ }^{2}$. In addition to unifying the analysis for different KPIs and different MIMO configurations, the presented model accounts for the effect of data retransmission upon decoding errors, in which the temporal correlation between the original and retransmission SINRs is captured. The developed framework relies on abstracting the unnecessary information conveyed within the interfering symbols by Gaussian signals along with a unified equivalent SISO-SINR representation for the different MIMO schemes. The Gaussian signaling approximation simplifies the decoding error analysis without sacrificing the model accuracy and aligns the decoding error analysis with the outage probability analysis. The Gaussian signaling abstraction is proposed in [29] for a SISO cellular network, which is generalized to MIMO cellular networks in this paper.

It is worth noting that the SISO-SINR representation used in this paper was used in the context of ad-hoc networks to study the outage probability and transmission capacity in [30]. In the context of cellular networks, it was also used to study OSTBC in [4], [31], [32], receive diversity in [5], and MU-MIMO in [14]. However, the analysis in [4], [5], [14], [31], [32] is confined to outage probability and ergodic rate only. Also, the OSTBC studies in [4], [31], [32] used the SISO-SINR model as an approximation for the outage probability characterization. In this paper, we extend the equivalent SISO-SINR model to evaluate decoding error probability, outage probability, ergodic rate, and throughput in MIMO cellular networks with transmit diversity (MISO), receive diversity (SIMO), OSTBC, MUMIMO, and spatial multiplexing with zero-forcing receivers (ZF-Rx). Furthermore, we prove that the equivalent SISOSINR model is exact in the case of OSTBC MIMO scheme.

To the best of our knowledge, this paper is the first to present such unified study that can be used to investigate the diversity-multiplexing tradeoffs between different MIMO configurations in terms of different performance metrics and provide guidelines for MIMO cellular network design. Particularly, we propose an automated reliable strategy to determine which MIMO settings to deploy in order to achieve a desired network objective under performance constraints. The main contributions of the developed framework can be

\footnotetext{
${ }^{2}$ This paper is an extension of the work reported in [28] and presented in
} IEEE International Conference on Communications (ICC), 2016. summarized in the following points:

- Developing a unified mathematical model that bridges the gap between error probability, outage probability, and ergodic rate analysis. Hence, it is possible to look at all three performance metrics within a single study.

- Providing simplified analysis and computational complexity reduction while maintaining the model accuracy, when compared to the exact analysis in [16].

- Accounting for the SINR temporal correlation and quantifying the resulting diversity loss.

- Revealing the multiplexing cost, in terms of outage probability and decoding error, in large-scale cellular networks. We also show the appropriate diversity compensation for such cost.

- Proposing a reliable network design strategy that is capable of appropriately adjusting the network parameters to meet desired design criteria.

\section{A. Organization \& Notation}

The paper is organized as follows. Section II presents the generic system model for a downlink cellular network deploying an arbitrary MIMO setup. In Section III the Gaussian signaling approximation and the equivalent SISOSINR model are presented. The unified model with and without retransmission is presented in Section IV. Section $\mathrm{V}$ illustrates how to represent different MIMO schemes via the equivalent SISO-SINR model. The model validation, via Monte-Carlo simulations, and the key findings of the paper are presented in Section VI and the paper is concluded in Section VII. Throughout the paper, we use the following notations: small-case bold-face letters $(\mathbf{x})$ denote column vectors, upper-case bold-face letters $(\mathbf{X})$ denote matrices, $(\cdot)^{T}$ and $(\cdot)^{H}$ denote the transnpose and Hermitian operators, respectively. $\|\cdot\|$ is the Euclidean norm operator, $\mathbb{E}_{x}[\cdot]$ and $\operatorname{Var}_{x}[\cdot]$ are the expectation and variance computed with respect to (w.r.t) the random variable $x$, respectively, and $\operatorname{erfc}(x)=\frac{2}{\sqrt{\pi}} \int_{x}^{\infty} e^{-t^{2}} \mathrm{~d} t$ is the complementary error function.

\section{System Model}

\section{A. Network and Propagation Models}

We consider a single-tier downlink ${ }^{3}$ cellular network, where the BSs locations are modeled by a homogeneous Poisson point process (PPP) $\Psi_{B}$ with intensity $\lambda_{B}$. UEs are distributed according to an independent homogeneous PPP $\Psi_{u}$ with intensity $\lambda_{u}$. BSs and UEs are equipped by $N_{t}$ and $N_{r}$ colocated antennas, ${ }^{4}$ respectively. Conditions on the relation between $N_{r}$ and $N_{t}$ depend on the MIMO setup under study, as will be shown later. Without loss of generality, we assume that $\Psi_{B}=\left\{r_{o}, r_{1}, r_{2}, \cdots\right\}$ contains the ascending ordered distances of the BSs from the origin (i.e., $r_{o}<r_{1}<r_{2}$ ) and that the analysis is conducted on a test user located at the origin [26], [35]. Assuming nearest BS association, the test user is subject to interference from the BSs in $\Psi^{o}=\Psi_{B} \backslash r_{o}$, in which the distance between the test user and its serving BS has the probability distribution

\footnotetext{
${ }^{3}$ The proposed analysis can be applicable to the uplink transmission with power control, following [19], [29], [33], [34].

${ }^{4}$ Colocated antennas is a common assumption in MIMO models based on stochastic geometry analysis to maintain the model tractability [4]-[8], [14]-[20].
} 
function (PDF) $f_{r_{o}}(r)=2 \pi \lambda_{B} r e^{-\pi \lambda_{B} r^{2}}, r_{o}>0$. Let $p$ be the independent transmission probability for each BS in $\Psi^{o}$, then, the point process of the active interfering BSs $\tilde{\Psi}^{o} \subseteq \Psi^{o}$ after independent thinning is also a PPP but with intensity $\lambda=p \lambda_{B}$ [35]. This assumption is used to reflect load awareness and/or frequency reuse as discussed in [16], [36]. Note that $p$ can be calculated as in [37], and setting $p=1$ gives the traditional saturation condition (i.e., $\lambda_{u} \gg \lambda$ ) where all BSs are active.

A distance-dependent power-law path-loss attenuation is employed, in which the signal power attenuates at the rate $r^{-\eta}$ with the distance $r$, where $\eta>2$ is the path-loss exponent. In addition to path-loss attenuation, we consider a Rayleigh multi-path fading environment between transmitting and receiving antennas, such that fading channels are independent from each other. That is, the channel gain matrix from a transmitting BS to a generic UE, denoted as $\mathbf{H} \in \mathbb{C}^{N_{r} \times N_{t}}$, has independent zero-mean unit variance complex Gaussian entries, such that $\mathbf{H} \sim \mathcal{C N}(0, \mathbf{I})$, where $\mathbf{I}$ is the identity matrix.

\section{B. Downlink MIMO Received Signal Model}

For a general MIMO setup in Rayleigh fading environment, with arbitrary precoding/combining schemes, the complex baseband received signal vector accounting for the precoding matrices is denoted as $\mathbf{y}$, and after applying combining matrices is expressed as $\tilde{\mathbf{y}}$, where

$$
\tilde{\mathbf{y}}=\sqrt{\frac{P}{r_{o}^{\eta}}} \mathbf{W}_{o} \mathbf{H}_{o} \mathbf{V}_{o} \mathbf{s}+\sum_{r_{i} \in \tilde{\Psi}^{o}} \sqrt{\frac{P}{r_{i}^{\eta}}} \mathbf{W}_{o} \mathbf{H}_{i} \mathbf{V}_{i} \mathbf{s}_{i}+\mathbf{W}_{o} \mathbf{n},
$$

where $P=\frac{E_{s}}{N_{+}}$is the transmit power per antenna at the BSs such that $E_{s}$ is the energy per symbol, $\mathbf{H}_{o} \in \mathbb{C}^{N_{r} \times N_{t}}$ is the useful channel matrix from the serving BS, and $\mathbf{H}_{i} \in \mathbb{C}^{N_{r} \times N_{t}}$ is the interfering channel matrix from the $i^{t h}$ interfering BS, $\mathbf{H}_{o}$ and $\mathbf{H}_{i}$, have independent and identically distributed (i.i.d.) $\mathcal{C N}(0,1)$ entries, $\mathbf{s} \in \mathbb{C}^{L \times 1}$ and $\mathbf{s}_{i} \in \mathbb{C}^{L \times 1}$ are, respectively, the intended and interfering symbols vector, where $L$ represents the number of multiplexed data streams ${ }^{5}$. The symbols in $\mathbf{s}$ and $\mathbf{s}_{i}$ are independently drawn from an equiprobable two dimensional unit-energy constellations. The matrices $\mathbf{V}_{o} \in \mathbb{C}^{N_{t} \times L}$ and $\mathbf{V}_{i} \in \mathbb{C}^{N_{t} \times L}$ are the intended and interfering precoding matrices at the intended BS and the $i^{\text {th }}$ interfering BS, respectively, while $\mathbf{W}_{o} \in \mathbb{C}^{L \times N_{r}}$ is the combining matrix at the test receiver. Note that $\mathbf{W}_{o}$ and $\mathbf{V}_{o}$ are tailored to each realization of $\mathbf{H}_{o}$ and are determined based on the employed MIMO scheme at the serving BS. On the other hand, $\mathbf{V}_{i}$ depends on the employed MIMO scheme at the $i^{\text {th }}$ interfering BS and is independent from the interfering channel matrix $\mathbf{H}_{i}$ as we assume no interBS interference management. $\mathbf{n} \in \mathbb{C}^{N_{r} \times 1}$ is the zero-mean additive white Gaussian noise vector with covariance matrix $\mathcal{N}_{o} \mathbf{I}_{N_{r}}$, where $\mathbf{I}_{N_{r}}$ is the identity matrix of size $N_{r}$. Last, we assume a per-symbol maximum likelihood (ML) receiver at the test UE to decode the symbols in $\mathbf{s}$.

\footnotetext{
${ }^{5}$ According to the employed MIMO setup, we might need to introduce a slight abuse of notation to preserve the convention used in (1). For instance, in multi-user MIMO setup with $\mathcal{K}$ single-antenna users, the parameter $N_{r}$ should be replaced with $\mathcal{K}$ so that the signal model in (1) remains valid.
}

\section{Gaussian Signaling APPROXIMATION \& EQUIVALENT SISO REPRESENTATION}

Assuming per-stream symbol-by-symbol ML receiver, the employed precoding, combining, and equalization techniques decouple symbols belonging to different streams (i.e., in case of multiplexing) and/or combine symbols belonging to the same stream (i.e., in case of diversity) at the decoder to allow disjoint and independent symbol detection across the multiplexed data streams. Hence, the precoding and combining matrices $\left(\mathbf{W}_{o}\right.$ and $\left.\mathbf{V}_{o}\right)$ are tailored to each realization of $\mathbf{H}_{o}$ such that the product $\mathbf{W}_{o} \mathbf{H}_{o} \mathbf{V}_{o}$ always gives a diagonal matrix of size $L$, with the appropriate, possibly different, diagonal values that correspond to the stream being decoded. Without loss of generality, let us focus on the decoding performance of a generic symbol in the $l^{\text {th }}$ stream, in which the instantaneous received signal after applying combining/precoding techniques is given by

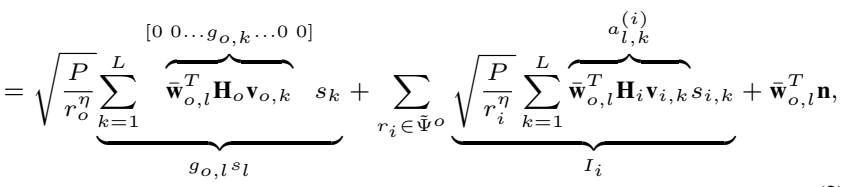

such that $l \in\{1, \cdots, L\}, \overline{\mathbf{w}}_{o, l}$ is the $l^{\text {th }}$ column of matrix $\mathbf{W}_{o}^{T}$, and $\mathbf{v}_{i, k}$ is the $k^{t h}$ column of matrix $\mathbf{V}_{i}$ which is designed based on the channel matrix between the $i^{\text {th }}$ interfering BS and its associated users, denoted as $\tilde{\mathbf{H}}_{i}$. Further, $g_{o, l}$ is a real random scaling factor that appears in the intended signal due to the equalization applied to detect the $l^{\text {th }}$ desired symbol, $a_{l, k}^{(i)}, \forall k$ are the complex random coefficients combining the interfering symbols from the antennas of the $i^{t h}$ interfering BS. As shown in (2), the coefficients $a_{l, k}^{(i)}$ are generated from the product $\overline{\mathbf{w}}_{o, l}^{T} \mathbf{H}_{i} \mathbf{v}_{i, k}$, which capture per-BS precoding and test receiver combining effects on the aggregate interference. As discussed earlier, $\overline{\mathbf{W}}_{o}$ and $\mathbf{V}_{i}$ are designed independently from each other and neither of them accounts for the realization of the interfering channel matrix $\mathbf{H}_{i}$. Therefore, the coefficients $a_{l, k}^{(i)}$ randomly change with each realization of $\mathbf{H}_{o}, \mathbf{H}_{i}$, and $\tilde{\mathbf{H}}_{i}$.

It is clear, from (2), that the aggregate interference seen at the decoder of the test UE is affected by the per-interfering BS precoding scheme $\left(\mathbf{v}_{i, k}\right)$, the number of streams transmitted by each BS $(L)$, the per-stream transmitted symbol $\left(s_{i, k}\right)$, and the employed combining technique $\left(\overline{\mathbf{w}}_{o, l}\right)$ at the test UE. Therefore, characterizing the aggregate interference in (2) is essential to characterize the MIMO operation in cellular networks. The aggregate interference term contains three main sources of randomness, namely, the network geometry, the channel gains ${ }^{6}$, and the interfering symbols. The average decoding error performance is only characterized for certain distributions of additive noise channels (e.g., Gaussian [38], Laplacian [39], [40], and Generalized Gaussian [41]), in which the additive white Gaussian noise (AWGN) channel represents the simplest case. Accounting for the exact distribution of the interfering symbols, the interference-plus-noise distribution does not directly fit into any of the distributions where the average decoding error performance is known. Hence, averaging over the transmitted symbols cannot be directly conducted unless the interference-plus-noise term is expressed or approximated via one of the distributions where the average decoding error performance is known. The authors in [16] proposed the equivalent-in-distribution (EiD)

\footnotetext{
${ }^{6}$ The precoding and combining matrices are functions of the channel gains.
} 
approach where an exact conditional Gaussian representation for the aggregate interference in (2) is achieved. Hence, the conditional error probability analysis, conditioned on network geometry and channel gains, is conducted via error probability expressions for AWGN channels, followed by a deconditioning step. The main drawback of the EiD approach is that it requires characterizing the interference signals at the baseband level to achieve the conditional Gaussian representation, which complicated both averaging steps, especially in MIMO networks. Furthermore, the EiD approach for the error probability analysis in [16] is disjoint from the outage probability and ergodic rate analysis in [4], [6]-[12], [14], [15], [19], [20].

To facilitate the error probability analysis and achieve a unified error probability, outage probability and ergodic rate analysis, we only account for the entries in the intended symbol vector $\mathbf{s}$ of (1) and abstract the entries in $\mathbf{s}_{i}$ by i.i.d. zero-mean Gaussian signals $\tilde{\mathbf{s}}_{i}$ with unit-variance. Such abstraction ignores the unnecessary and usually unavailable information of the interfering signals. Assuming Gaussian signaling for the interfering symbols (i.e., the entries in $\tilde{\mathbf{s}}_{i}$ are Gaussian), (2) can be rewritten as

$$
\tilde{y}_{l}=\sqrt{\frac{P}{r_{o}^{\eta}}} g_{o, l} s_{l}+\sum_{r_{i} \in \tilde{\Psi}^{o}} \underbrace{\sqrt{\frac{P}{r_{i}^{\eta}} \sum_{k=1}^{L} a_{l, k}^{(i)} \tilde{s}_{i, k}}}_{I_{i}}+\overline{\mathbf{w}}_{o, l}^{T} \mathbf{n} .
$$

It is important to note that the random variables $g_{o, l} \forall l$ are i.i.d, and hence, there is no loss in generality to drop the index $l$ and study an arbitrary stream. Conditioned on $r_{i}$ and $a_{l, k}^{(i)} \forall\{i, k, l\}$, the lumped interference-plus-noise term in (3) is Gaussian because of the Gaussianity of $\tilde{s}_{i, k}, \forall i, k$. This renders the well-known AWGN error probability expressions legitimate to conduct the averaging over the transmitted symbols, in which the noise variance used in the AWGNbased expressions is replaced by the variance of the lumped interference and noise terms in (3). The Gaussian signaling approximation leads to the following proposition.

Proposition 1: Consider a downlink MIMO cellular network with $N_{t}$ antennas at each BS and $N_{r}$ antennas at each UE in a Rayleigh fading environment with i.i.d. unitmean channel power gains and Gaussian signaling approximation for the interfering symbols, then the per-data stream conditional SINR at the decoder of a generic UE after combining/equalization can be represented via the following equivalent SISO-SINR

$$
\Upsilon=\frac{\operatorname{Pr}_{o}^{-\eta} \tilde{g}_{o}}{\sum_{r_{i} \in \tilde{\Psi}^{o}} \operatorname{Pr}_{i}^{-\eta} \tilde{g}_{i}+\mathcal{N}_{o}},
$$

where the random variables $\tilde{g}_{o} \sim \operatorname{Gamma}\left(m_{o}, 1\right)$ and $\tilde{g}_{i} \sim \operatorname{Gamma}\left(m_{i}, 1\right)$ capture the effect of MIMO precoding, combining, and equalization. The values of $m_{o}$ and $m_{i}$ are determined based on the number of antennas $\left(N_{t}\right.$ and $\left.N_{r}\right)$, the number of multiplexed data streams per BS $(L)$ and the employed MIMO configuration as shown in Table I.

Proof: The detailed discussion and proof of each MIMO setup is given in Section $\mathrm{V}$ in the corresponding lemma shown in Table I. Here, we just sketch a high-level proof of the proposition. The equivalent channel gains in (4) are $\tilde{g}_{o}=\left|g_{o}\right|^{2}$ and $\tilde{g}_{i}=\left|g_{i}\right|^{2}=\sum_{k=1}^{L}\left|a_{l, k}^{(i)}\right|^{2}$, where $g_{o}$ is the random scale for the intended symbol due to precoding and combining/equalization as show in (2). Since precoding and combining/equalization are usually in the form of linear combination of the channel power gains and that the channel gains have independent Gaussian distributions, both random variables $\tilde{g}_{o}$ and $\tilde{g}_{i} \forall i$ are independent $\chi^{2}$-distributed with degrees of freedom equal to the number of linearly combined random variables, which depends on the number of antennas, precoding technique, and number of multiplexed data streams per BS. Note that the $\chi^{2}$ distribution for interfering channel gains $\tilde{g}_{i}$ is exact only if the precoding vectors in each BS are independent. In the case of dependent precoding vectors, the correlation is ignored and the $\chi^{2}$ distribution for $\tilde{g}_{i}$ is an approximation. Such approximation is commonly used in the literature for tractability [4], [6]-[11], [14]-[17], and is verified in Section VI. Exploiting the one-to-one mapping between the $\chi^{2}$ distribution and the gamma distribution, we follow the convention in [4], [6]-[11], [14]-[17] and use the gamma distribution, instead of the $\chi^{2}$ distribution, for $\tilde{g}_{o}$ and $\tilde{g}_{i}$.

It is important to note that, the proposed SISO-SINR model relies on the assumption of independent Rayleigh fading channels. While spatially correlated fading channels model provides more realistic fading environment [42], however, such correlation is ignored for tractability as in [4], [6]-[11], [14]-[17]. Proposition 1 gives the equivalent SISO representation for the MIMO cellular network in which the effect of precoding, combining, and equalization of the employed MIMO scheme is abstracted by the random variables $\tilde{g}_{o}$ and $\tilde{g}_{i}, \forall i$ in (4). Hence, unified analysis and expressions for different KPIs and MIMO configurations, respectively, are viable as shown in the next section.

\section{UNIFIED PERFORMANCE ANALYSIS}

Based on the Gaussian signaling approximation, interference-plus-noise in (3) is conditionally Gaussian. Hence, the decoding error performance of the MIMO scheme is studied by plugging the conditional SINR in (4) with the appropriate channel gains (i.e., $\tilde{g}_{o}$ and $\tilde{g}_{i}$ ) in the corresponding AWGN-based decoding error expression, followed by an averaging over the channel gains and network geometry. Using the AWGN expression for the SEP for square quadrature amplitude modulation ( $M$-QAM) scheme given in [38], the ASEP in MIMO cellular networks can be expressed as

$$
\operatorname{ASEP}(\Upsilon)=w_{1} \mathbb{E}[\operatorname{erfc}(\sqrt{\beta \Upsilon})]+w_{2} \mathbb{E}\left[\operatorname{erfc}^{2}(\sqrt{\beta \Upsilon})\right]
$$

where $w_{1}=2 \frac{\sqrt{M}-1}{\sqrt{M}}, w_{2}=-\left(\frac{\sqrt{M}-1}{\sqrt{M}}\right)^{2}$, and $\beta=\frac{3}{2(M-1)}$ are constellation-size specific constants ${ }^{7}$. The Gaussian signaling approximation is also the key that unifies the ASEP, outage probability, and ergodic rate analysis. This is because both outage and capacity are information theoretic KPIs that implicitly assume Gaussian codebooks, which directly lead to the conditional SINR in the form given by Proposition 1 . Consequently, both the outage probability and ergodic capacity are also functions of the SINR in the form of (4), and are given by

$$
\mathcal{O}=\mathbb{P}\{\Upsilon<\theta\},
$$

\footnotetext{
${ }^{7}$ The error probability expression in (5) can model other modulation schemes by just changing the modulation-specific parameters as shown in [38].
} 


\begin{tabular}{|c|c|c|c|c|c|}
\hline MIMO Setup & $L$ & $\boldsymbol{m}_{\boldsymbol{o}}$ & $\boldsymbol{m}_{\boldsymbol{i}}$ & Accuracy & Proof \\
\hline \hline SIMO & 1 & $N_{r}$ & 1 & Exact & Lemma 3 \\
\hline OSTBC & $N_{s}$ & $N_{s} N_{r}$ & $N_{s}$ & Exact & Lemma 4 \\
\hline ZF-Rx & $N_{t}$ & $N_{r}-N_{t}+1$ & $N_{t}$ & Exact & Lemma 5 \\
\hline SDMA & $\mathcal{K}$ & $N_{t}-\mathcal{K}+1$ & $\mathcal{K}$ & Approx. & Lemma 6 \\
\hline MISO & 1 & $N_{t}$ & 1 & Exact & Corollary 1 \\
\hline SM-MIMO & $N_{t}$ & $N_{r}$ & $N_{t}$ & Approx. & Lemma 7 \\
\hline
\end{tabular}

TABLE I: SISO-equivalent gamma distribution parameters for various MIMO settings.

and

$$
\mathcal{R}=\mathbb{E}[\ln (1+\Upsilon)]
$$

In order to derive the actual performance metrics from the conditional SINR expression in Section III, the expectations in (5), (6), and (7) are w.r.t the network geometry and channel gains, which are evaluated via stochastic geometry analysis. Such expectations are usually expressed in terms of the Laplace transform (LT) ${ }^{8}$ of the aggregate interference power in (4), denoted as $\mathcal{I}=\sum_{r_{i} \in \tilde{\Psi}^{o}} \operatorname{Pr}_{i}^{-\eta} \tilde{g}_{i}$. The LT of the interference power in the SISO-equivalent SINR given in (4) is characterized by the following lemma.

Lemma 1: Consider a cellular network with MIMO transmission scheme that can be represented via the equivalent SISO-SINR in (4) and BSs modeled via a PPP with intensity $\lambda$, in which each BS transmits a symbol vector of length $L$ per channel use (pcu) with symbols drawn from a zeromean unit-variance Gaussian distribution, then the LT of the interference power affecting an arbitrary symbol at a receiver located $r_{o}$ meters away from its serving BS is given by

$\mathcal{L}_{\mathcal{I} \mid r_{o}}(z)=\exp \left\{-\pi \lambda r_{o}^{2}\left[\left({ }_{2} F_{1}\left(\frac{-1}{b}, m_{i} ; 1-\frac{1}{b} ;-z P r_{o}^{-\eta}\right)-1\right)\right]\right\}$,

where ${ }_{2} F_{1}(\cdot, \cdot ; \cdot ; \cdot)$ is the Gauss hypergeometric function [43].

Proof: Starting from the definition of the LT, we have

$$
\begin{aligned}
\mathcal{L}_{\mathcal{I} \mid r_{o}}(z)= & \mathbb{E}\left[\exp \left\{-z \sum_{r_{i} \in \tilde{\Psi}^{o}} \operatorname{Pr}_{i}^{-\eta} \tilde{g}_{i}\right\}\right] \\
& \stackrel{(a)}{=} \exp \left\{-2 \pi \lambda \int_{r_{o}}^{\infty} \mathbb{E}\left[1-e^{-z P x^{-\eta} \tilde{g}}\right] x \mathrm{~d} x\right\} \\
& \stackrel{(b)}{=} \exp \left\{-2 \pi \lambda \int_{r_{o}}^{\infty} \mathbb{E}\left[1-\frac{1}{\left(1+z P x^{-\eta}\right)^{m_{i}}}\right] x \mathrm{~d} x\right\},
\end{aligned}
$$

where $(a)$ follows from the probability generating functional (PGFL) of the homogeneous PPP [35] constituted by the interferers lying outside a disk of radius $r_{o}$, i.e. $r_{i}>r_{o}$, with intensity $\lambda$, and $(b)$ follows from the LT of the gamma distributed channel gains with shape parameter $m_{i}$ and unity scale parameter. Solving the integral, yields the LT in (8).

Using Proposition 1 and Lemma 1, we arrive to the unified MIMO expressions for the ASEP, outage probability, and ergodic rate in the following theorem.

Theorem 1: Unified Analysis: Consider a cellular network with MIMO transmission scheme that can be represented via the equivalent SISO-SINR in (4) and BSs modeled via a PPP with intensity $\lambda$, in which each BS transmits a symbol vector of length $L$ pcu with symbols drawn from an equiprobable unit-power $M$-QAM modulation scheme, then the ASEP for an arbitrary symbol is approximated by $(10)$, where ${ }_{1} F_{1}(\cdot ; \cdot ; \cdot)$ is the Kummer confluent hypergeometric function [43].

\footnotetext{
${ }^{8}$ The LT of the interference is a short for the LT of the probability density function (PDF) of the interference, which is equivalent to the moment generating function but with negative argument.
}

For an interference-limited scenario, the probability that the average SIR (averaged over all symbols) for an arbitrary stream goes below a threshold $\theta$ is given by

$\mathcal{O}(\theta)=1-\left.\int_{0}^{\infty} 2 \pi \lambda_{B} x e^{-\pi \lambda_{B} x^{2}} \sum_{j=0}^{m_{o}-1} \frac{(-1)^{j}}{j !} \frac{\mathrm{d}^{j}}{\mathrm{~d} z^{j}}\left(\frac{\theta x^{\eta}}{P}\right)^{j} \mathcal{L}_{\mathcal{I} \mid x}(z)\right|_{z=\frac{\theta x^{\eta}}{P}} \mathrm{~d} x$,

and the ergodic rate for an arbitrary data stream is given by

$\mathcal{R}=\int_{0}^{\infty} \int_{0}^{\infty} 2 \pi \lambda_{B} x e^{-\pi \lambda_{B} x^{2}} \mathcal{L}_{\mathcal{I} \mid x}\left(\frac{z x^{\eta}}{P}\right)\left(\frac{1-(1+z)^{-m_{o}}}{z}\right) \mathrm{d} z \mathrm{~d} x$,

where $\mathcal{L}_{\mathcal{I} \mid x}(z)$ in (10), (11), and (12) is the LT given in Lemma 1 when replacing $r_{o}$ with $x$.

Proof: See Appendix A.

The ASEP given in (10) is an approximation due to the Gaussian signaling abstraction used in (3). On the other hand, expressions for the outage probability and ergodic rate are exact, because both are typically derived in the literature based on the Gaussian codebooks assumption. The outage probability in (11) is given for interference-limited networks for tractability, which is a common assumption in cellular network because the interference term usually dominates the noise. Both equations (11) and (12) are approximations in cases of SDMA and SM-MIMO due to the approximate estimation of the interference as shown in Table I. It is ought to be mentioned that different exact/approximate forms for the outage probability and ergodic rate, shown in (11) and (12), respectively, can be derived via Gil-Pelaez inversion theorem as in [25], [44] and Alzer's inequality as in [45], [46]. Nevertheless, both approaches are based on the Gaussian signaling approximation and equivalent SISO representation given in Proposition 1.

The ASEP expression given in (10) presents three advantages over the ASEP expressions given in [16]. First, (10) provides a unified ASEP expression for all considered MIMO schemes. Second, the ASEP is characterized based on the LT given in Lemma 1, which is the same LT used for characterizing the outage probability and ergodic rate. Third, the computational complexity to evaluate (10) is less than the complexity of the ASEP expressions in [16]. The reduced complexity of (10) is because it includes a single hypergeometric function in the exponential term while the expressions for the ASEP in [16] include summations of hypergeometric functions inside the exponential term. It should be highlighted that the EiD approach leads to the same computational complexity as in (10) for some modulation schemes, e.g., phase-shift-keying ( $M$-PSK) [16]. Nevertheless, the proposed Gaussian approximation and equivalent SISO representation simplifies the ASEP analysis and unifies it with outage and ergodic rate results when compared to the EiD approach which always involves the complex baseband interference analysis.

\section{A. The Effect of Temporal Correlation on Retransmissions}

The network performance with retransmission cannot be directly deduced from Lemma 1 and Theorem 1 due to the temporal interference correlation. Despite that we assume that the channel fading independently changes from one time slot to another, the interference at a given location is correlated across time for the same network realization due to the fixed locations of the complete set of BSs. In other 


$$
\begin{aligned}
\mathrm{ASEP} & \approx w_{1}\left[1-\frac{\Gamma\left(m_{o}+\frac{1}{2}\right)}{\Gamma\left(m_{o}\right)} \frac{2}{\pi} \int_{0}^{\infty} \int_{0}^{\infty} 2 \pi \lambda_{B} x e^{-\pi \lambda_{B} x^{2}} \frac{1}{\sqrt{z}} e^{-z\left(1+\frac{m_{o} \mathcal{N}_{o} x^{\eta}}{\beta P}\right)}{ }_{1} F_{1}\left(1-m_{o} ; \frac{3}{2} ; z\right) \cdot \mathcal{L}_{\mathcal{I} \mid x}\left(\frac{m_{o} z x^{\eta}}{\beta P}\right) \mathrm{d} x \mathrm{~d} z\right] \\
& +w_{2}\left[1-\frac{4 m_{o}}{\pi} \int_{0}^{\infty} \int_{0}^{\infty} 2 \pi \lambda_{B} x e^{-\pi \lambda_{B} x^{2}} e^{-z\left(\frac{m_{o} \mathcal{N}_{O} x^{\eta}}{\beta P}\right)} \int_{0}^{\frac{\pi}{4}}{ }_{1} F_{1}\left(m_{o}+1 ; 2 ; \frac{-z}{\sin ^{2} \vartheta}\right) \frac{1}{\sin ^{2} \vartheta} \cdot \mathcal{L}_{\mathcal{I} \mid x}\left(\frac{m_{o} z x^{\eta}}{\beta P}\right) \mathrm{d} \vartheta \mathrm{d} x \mathrm{~d} z\right]
\end{aligned}
$$

words, assuming a static UE, a subset of the interferers at a given time slot might also be interfering in subsequent time slots, which introduces temporal interference correlation that needs to be taken into account. In this section, we study temporal correlation between two arbitrary time slots at the same spatial location. Based on the interference temporal correlation, we derive the conditional success probability of a retransmission such that an earlier transmission at the same location was unsuccessful. Note that the presented analysis captures the pairwise temporal correlation across any two time slots as long as the spatial location of the test receiver is fixed. Extensions to more than two time slots is cumbersome and is not considered in this paper. To incorporate the effect of retransmission into the analysis, the temporal correlation of the interference should be characterized via the joint LT of the interferences across different time slots, as given by the following lemma.

Lemma 2: Consider a cellular network with MIMO transmission scheme that can be represented via the equivalent SISO-SINR in (4) and BSs modeled via a PPP with intensity $\lambda$ and activity factor $p$, the joint LT of the interferences at a given location at two different time slots, denoted by $\mathcal{I}_{1}$ and $\mathcal{I}_{2}$, such that the interfering BSs may use different MIMO scheme across time, is given by (13), where $m_{i, 1}$ and $m_{i, 2}$ are the rates of the Gamma distributed equivalent channel gains (given in Table I) corresponding to the employed MIMO scheme at the first and second time slots, respectively, and $\mathcal{F}_{1}(\cdot ; \cdot, \cdot ; \cdot ; x, y)$ is the Appell Hypergeometric function, which extends the hypergeometric function to two variables $x$ and $y$ [47].

Proof: See Appendix B

The average coverage probability (defined as $1-\mathcal{O}(\theta)$ ) with retransmissions and independent signal decoding is given by

$\mathcal{P}_{c}(\theta)=\mathbb{P}\left(\bar{\Upsilon}_{1}>\theta\right)+\mathbb{P}\left(\bar{\Upsilon}_{2}>\theta\right)-\mathbb{P}\left(\bar{\Upsilon}_{1}>\theta, \bar{\Upsilon}_{2}>\theta\right)$

where $\bar{\Upsilon}_{1}$ and $\bar{\Upsilon}_{2}$ are the SIRs at the first and second transmissions. Using the joint LT in Lemma 2, the average coverage probability with retransmission in MIMO cellular network is given by the following theorem.

Theorem 2: Consider a cellular network with MIMO transmission scheme that can be represented via the equivalent SISO-SINR in (4) and BSs modeled via a PPP with intensity $\lambda$, the SIR coverage probability for a generic UE with retransmission such that the serving BS and interfering BSs may use different MIMO schemes across time, is given by (15),

$$
\text { where } \bar{\theta}=\frac{\theta x^{\eta}}{P} \text {. }
$$

Proof: See Appendix C

Before giving numerical results and insights obtained from the developed mathematical model, we first illustrate how the equivalent SISO-SINR model given in Proposition 1 holds for the considered MIMO schemes.

\section{Characterizing MiMO CONFIGURATIONS}

This section details the methodology to abstract different MIMO configuration via the equivalent SISO model given in Proposition 1 with parameters given in Table I. In order to conduct the analysis for the different MIMO setups, we first need to define the set $\{\breve{\mathbf{H}}\}$ as the set of channel matrices that affect the aggregate interference signals due to precoding and/or combining. For instance, due to precoding, combining, and equalization, the interference from the $i^{\text {th }}$ interfering BS is multiplied by $\mathbf{W}_{o} \mathbf{H}_{i} \mathbf{V}_{i}$, and hence, $\{\breve{\mathbf{H}}\}=\left\{\mathbf{H}_{o}, \tilde{\mathbf{H}}_{i}\right\}$, where $\mathbf{H}_{o}$, and $\tilde{\mathbf{H}}_{i}$ are the channel matrices between, respectively, the intended BS and the test user, and the $i^{\text {th }}$ interfering BSs and its associated users. The methodology to characterize the distribution of the equivalent channel gains are given in the following steps:

1) SNR characterization: $\tilde{g}_{o}$ is first characterized by projecting the signal of the intended data-stream on the null-space of the signals of the other data streams that are multiplexed by the intended BSs. Note that we may manipulate the resultant SNR such that the noise variance is not affected by any random variable as in (4) and the projection effect is contained in $\tilde{g}_{o}$ and $\tilde{g}_{i}$ only.

2) Per-stream equivalent channel gain representation: $\tilde{g}_{i}$ from each interfering BS is characterized based on the manipulation done in the SNR characterization in the previous step and characterizing $\left|a_{l, k}^{(i)}\right|^{2}$ given in (3). Note that $\left|a_{l, k}^{(i)}\right|^{2}$ is characterized based on $\{\breve{\mathbf{H}}\}$ which captures the channel gain matrices involved in precoding the signal at the $i^{t h} \mathrm{BS}$ and combining the interfering symbols at the test UE.

Based on the aforementioned two steps, the equivalent SISO-SINR given in Proposition 1 for the MIMO schemes given in Table I is illustrated in this section.

1) Single-Input-Multiple-Output (SIMO) systems: for a SIMO system, receive diversity is achieved using one transmit antenna (i.e., $L=N_{t}=1$ ) and $N_{r}$ receive antennas. Since $N_{t}=1$, then the intended and interfering channel vectors are denoted by $\mathbf{h}_{o}$ and $\mathbf{h}_{i} \in \mathbb{C}^{N_{r} \times 1}$, respectively. By employing Maximum Ratio-Combining (MRC) strategy to combine the received signals, then $\overline{\mathbf{w}}_{o}^{T}=\mathbf{h}_{o}^{H}$. The equivalent SISO channel gains are given by the following lemma.

Lemma 3: For a receive diversity SIMO setup technique, the Gamma distribution parameters for the equivalent intended and interfering channel gains are given by $\boldsymbol{m}_{\boldsymbol{o}}=\boldsymbol{N}_{\boldsymbol{r}}$ and $\boldsymbol{m}_{\boldsymbol{i}}=\mathbf{1}$, respectively.

Proof: See Appendix D

2) Orthogonal Space-Time Block Coding (OSTBC): Let a balanced orthogonal space-time block codes be transmitted over $T$ time instants such that only $N_{s} \leq N_{t}$ transmit antennas are active per time instant. The received signal is equalized via the equalizer $\mathbf{W}_{o}=\frac{\mathbf{H}_{\text {eff }}^{H}}{\left\|\mathbf{H}_{o}\right\|_{\mathrm{F}}}$, where $\mathbf{H}_{\mathrm{eff}}$ is the effective intended channel matrix depending on the employed 


$$
\begin{aligned}
& \mathcal{L}_{\mathcal{I}_{1}, \mathcal{I}_{2} \mid r_{o}}\left(z_{1}, z_{2}\right)= \exp \left\{-\pi \lambda r_{o}^{2}\left[p\left(\mathcal{F}_{1}\left(\frac{-2}{\eta} ; m_{i, 1}, m_{i, 2} ; 1-\frac{2}{\eta} ;-z_{1} r_{o}^{-\eta},-z_{2} r_{o}^{-\eta}\right)+1\right)\right.\right. \\
&\left.\left.+(1-p)\left({ }_{2} F_{1}\left(\frac{-1}{b}, m_{i, 2} ; 1-\frac{1}{b} ;-z P r_{o}^{-\eta}\right)+{ }_{2} F_{1}\left(\frac{-1}{b}, m_{i, 1} ; 1-\frac{1}{b} ;-z P r_{o}^{-\eta}\right)\right)-2\right]\right\} . \\
& \mathcal{P}_{c}(\theta)=\int_{0}^{\infty} 2 \pi \lambda_{B} x e^{-\pi \lambda_{B} x^{2}}\left[\left.\sum_{j_{1}=0}^{\left[m_{o, 1}-1\right.} \frac{(-1)^{j_{1}}}{j_{1} !} \bar{\theta}^{j_{1}} \frac{\partial^{j_{1}}}{\partial z_{1}^{j_{1}}} \mathcal{L}_{\mathcal{I}_{1} \mid x}\left(z_{1}\right)\right|_{z_{1}=\bar{\theta}}+\left.\sum_{j_{2}=0}^{m_{o, 2}-1} \frac{(-1)^{j_{2}}}{j_{2} !} \bar{\theta}^{j_{2}} \frac{\partial^{j_{2}}}{\partial z_{2}^{j_{2}}} \mathcal{L}_{\mathcal{I}_{2} \mid x}\left(z_{2}\right)\right|_{z_{2}=\bar{\theta}}\right. \\
&\left.-\left.\sum_{j_{1}=0}^{m_{o, 1}-1} \sum_{j_{2}=0}^{m_{o, 2}-1} \frac{(-1)^{j_{1}+j_{2}}}{j_{1} ! j_{2} !} \bar{\theta}^{j_{1}+j_{2}} \frac{\partial^{\left(j_{1}+j_{2}\right)}}{\partial z_{1}^{j_{1}} \partial z_{2}^{j_{2}}} \mathcal{L}_{\mathcal{I}_{1}, \mathcal{I}_{2} \mid x}\left(z_{1}, z_{2}\right)\right|_{z_{1}=z_{2}=\bar{\theta}}\right] \mathrm{d} x .
\end{aligned}
$$

orthogonal code [4], [16]. Since no precoding is applied then $\mathbf{V}_{o}=\mathbf{V}_{i}=\mathbf{I}_{N_{t}}$. The equivalent SISO channel gains are given by the following lemma.

Lemma 4: A space-time encoder is employed at the network BSs. Then, the Gamma distribution parameters are given as $\boldsymbol{m}_{\boldsymbol{o}}=\boldsymbol{N}_{\boldsymbol{s}} \boldsymbol{N}_{\boldsymbol{r}}$ and $\boldsymbol{m}_{\boldsymbol{i}}=\boldsymbol{N}_{\boldsymbol{s}}$.

\section{Proof: See Appendix D}

3) Zero-Forcing beamforming with ML Receiver (ZF$\boldsymbol{R} \boldsymbol{x})$ : ZF is a low-complexity suboptimal, yet efficient, technique to suppress interference from other transmitted symbols in the network. In order to recover the distinct transmitted streams, the received signal is multiplied by the equalizing matrix $\mathbf{W}_{o}=\left(\mathbf{H}_{o}^{H} \mathbf{H}_{o}\right)^{-1} \mathbf{H}_{o}^{H}$ representing the pseudoinverse of the intended channel matrix $\mathbf{H}_{o}$, whereas we assume no precoding at the transmitters side, i.e., $\mathbf{V}_{o}=\mathbf{V}_{i}=$ $\mathbf{I}_{N_{t}}$. The equivalent SISO channel gains are given by the following lemma.

Lemma 5: By employing a ZF-Rx such that $L=N_{t}$ distinct streams are being transmitted from the BSs, it can be shown that $m_{o}=N_{r}-N_{t}+1$ and $m_{i}=N_{t}$.

\section{Proof: See Appendix D}

4) Space-Division Multiple Access (SDMA): SDMA is used to accommodate more users on the same resources to increase the network capacity. In this case, we consider that each BS is equipped by $N_{t}$ transmit antennas and applies ZF transmission to simultaneously serve $\mathcal{K}$ single-antenna UEs that are independently and randomly distributed within its coverage area. To avoid rank-deficiency, we let $N_{t} \geq \mathcal{K}$, and hence, the number of data streams $L=\mathcal{K}$. A ZF-precoding in the form of $\mathbf{V}_{o}=\left[\mathbf{v}_{1}, \mathbf{v}_{2} \cdots \mathbf{v}_{\mathcal{K}}\right]$ such that $\mathbf{v}_{l}=\frac{\mathbf{q}_{l}}{\left\|\mathbf{q}_{l}\right\|}$ and $\mathbf{q}_{l}$ is defined as the $l^{\text {th }}$ column of $\mathbf{Q}=\mathbf{H}_{o}^{H}\left(\mathbf{H}_{o} \mathbf{H}_{o}^{H}\right)^{-1}$ is applied by the test $\mathrm{BS}$ and no combining is applied at the single antenna test UE, and hence, $\mathbf{W}_{o}=\mathbf{I}_{\mathcal{K}}$. The interfering BSs apply the same precoding and combining strategy, and hence, the interfering precoding matrices are in the form $\mathbf{V}_{i}=\left[\mathbf{v}_{i, 1}, \mathbf{v}_{i, 2} \cdots \mathbf{v}_{i, \mathcal{K}}\right]$ such that $\mathbf{v}_{i, k}=\frac{\mathbf{q}_{k}}{\left\|\mathbf{q}_{k}\right\|}$ and $\mathbf{q}_{k}$ is the $k^{t h}$ column of $\mathbf{Q}_{i}=\tilde{\mathbf{H}}_{i}^{H}\left(\tilde{\mathbf{H}}_{i} \tilde{\mathbf{H}}_{i}^{H}\right)^{-1}$, note that $\tilde{\mathbf{H}}_{i} \neq \mathbf{H}_{i}$ is the interfering channel matrix towards the corresponding intended users. The equivalent SISO channel gains are given by the following lemma.

Lemma 6: In a multi-user MIMO setup, the corresponding Gamma distributions parameters are given by $\boldsymbol{m}_{\boldsymbol{o}}=\boldsymbol{N}_{\boldsymbol{t}}-\mathcal{K}+\mathbf{1}$, and $\boldsymbol{m}_{\boldsymbol{i}} \approx \mathcal{K}$.

\section{Proof: See Appendix D}

Corollary 1: Single-User Beamforming (SU-BF):

The SDMA scenario reduces to SU-MISO (i.e., transmit diversity) setting if the number of served users in the network is $\mathcal{K}=1$. Hence, $m_{o}=N_{t}$ and $m_{i}=1$.

5) Spatially Multiplexed MIMO (SM-MIMO) systems:

for the sake of completeness, we also consider a spatially multiplexed MIMO setup with optimum joint maximum likelihood receiver. This case is important because it represents the benchmark for $\mathrm{ZF}$ decoding. Note that the analysis in this case is slightly different from the aforementioned schemes since joint detection is employed. Nevertheless, it can be represented via the equivalent SISO-SINR given in Proposition 1. Due to joint detection, no precoding/combining is applied such that $\mathbf{W}_{o}=\mathbf{V}_{o}=\mathbf{V}_{i}=\mathbf{I}_{N_{t}}$. To analyze this case, we define the error vector $\mathbf{e}(\mathbf{s}, \hat{\mathbf{s}})=\mathbf{s}-\hat{\mathbf{s}}$ as the distance between $\mathbf{s}$ and $\hat{\mathbf{s}}$ and hence we derive the APEP, which is then used to approximate the ASEP as shown in the following lemma.

Lemma 7: For a SM-MIMO transmission, the Gamma distribution parameter for the equivalent intended channel gains is given by $\boldsymbol{m}_{o}=N_{r}$, while for the equivalent interfering links is given by $\boldsymbol{m}_{\boldsymbol{i}}=\boldsymbol{N}_{\boldsymbol{t}}$. Furthermore, the averaged PEP over the distance distribution of $r_{o}$ is

$$
\begin{aligned}
\operatorname{APEP}(\|\mathbf{e}\|) & \approx 1-\frac{\Gamma\left(m_{o}+\frac{1}{2}\right)}{\Gamma\left(m_{o}\right)} \frac{2}{\pi} \int_{0}^{\infty} \int_{0}^{\infty} 2 \pi \lambda_{B} x e^{-\pi \lambda_{B} x^{2}} \\
& \times \frac{1}{\sqrt{z}} e^{-z\left(1+\frac{m_{o} \mathcal{N}_{o} x^{\eta}}{4\|\mathbf{e}\|^{2} P}\right)}{ }_{1} F_{1}\left(1-m_{o} ; \frac{3}{2} ; z\right) \\
& \times \mathcal{L}_{\mathcal{I} \mid x}\left(\frac{m_{o} z}{4\|\mathbf{e}\|^{2} P x^{-\eta}}\right) \mathrm{d} x \mathrm{~d} z
\end{aligned}
$$

Consequently, using the Nearest Neighbor approximation [48], where there are $M$ equiprobable symbols, then

$$
\operatorname{ASEP} \approx N_{\|\mathbf{e}\|_{\min }} \operatorname{APEP}\left(\|\mathbf{e}\|_{\min }\right)
$$

where $N_{\|\mathbf{e}\|_{\min }}$ is the number of constellation points having the minimum Euclidean distance denoted by $\min _{\mathbf{s}, \hat{\mathbf{s}}}\|\mathbf{e}(\mathbf{s}, \hat{\mathbf{s}})\|$ among all possible pairs of transmitted symbols, and hence is a modulation-specific parameter.

\section{Proof: See Appendix D}

\section{Numerical AND Simulation Results}

In this section, we verify the validity and accuracy of the proposed unified model and discuss the potential of such unified framework for designing cellular networks. The simulations setup is as follows. The BSs transmit powers $(P)$ vary while $\mathcal{N}_{o}$ is kept constant to vary the transmit SNR, the path-loss exponent $\eta=4$, the noise power $\mathcal{N}_{o}=-90 \mathrm{dBm}$, the BSs intensity $\lambda_{B}=10 \mathrm{BSs} / \mathrm{km}^{2}, \lambda_{u}=20$ users $/ \mathrm{km}^{2}$, and finally the activity factor $p=1$. The transmitted symbols are modulated using $M$-QAM modulation scheme. 


\section{A. Proposed model validation}

We validate Theorem 1 for the derived ASEP and outage probability expressions via Monte-Carlo simulations, in Fig. 1 , for a fixed number of antennas in order to seek a fair comparison among the MIMO schemes. That is, we consider different MIMO configurations with $N_{r}=2$ and $N_{t}=2$. Note that, for SIMO and MISO, $N_{t}$ and $N_{r}$ are set to 1 , respectively. Further, in SDMA scenario, the number of single-antenna users served in the network is $\mathcal{K}=2$. The figure verifies the accuracy of the Gaussian signaling approximation and the developed ASEP and outage probability model, in which the analytic expressions perfectly match the simulations.

Fig. 2 validates Theorem 2 for the outage before and after retransmission against Monte-Carlo simulations. Fig. 2(a) shows the time diversity loss due to interference temporal correlation when compared to the independent interference scenario. The figure shows that assuming independent interference across time is too optimistic, since those UEs requiring retransmissions are then biased to ones where there are interferers nearby due to interference temporal correlation. Nevertheless, it is possible for the network operators to exploit more diversity in the second transmission to compensate for the expected degraded retransmission performance. Fig. 2(b) shows the effect of incremental diversity in the second transmission on the outage performance for $m_{o}=2$ and $m_{i}=2$. The figure shows that adjusting the MIMO configuration such that $m_{o}=5$ in the retransmission compensates for the temporal correlation effect and achieves the same performance as independent transmission (e.g., up to $3 \mathrm{~dB}$ SIR improvement can be achieved).

\begin{tabular}{|c|c|c|c|cc|}
\hline MIMO Setup & $\boldsymbol{m}_{\boldsymbol{o}}$ & $\boldsymbol{m}_{\boldsymbol{i}}$ & $\begin{array}{c}\text { Ergodic Rate } \\
\text { (bits/sec/Hz) }\end{array}$ & \multicolumn{2}{|c|}{ No. of bits pcu } \\
& & & 16 -QAM & 16-1772 \\
\hline \hline SIMO & 2 & 1 & 2.9523 & 1.6926 & 2.1772 \\
\hline OSTBC & 4 & 2 & 2.9771 & 1.7228 & 2.2044 \\
\hline ZF-Rx & 1 & 2 & 3.1644 & 2.6300 & 2.7008 \\
\hline SDMA & 1 & 2 & 3.1644 & 2.6300 & 2.7008 \\
\hline MISO & 2 & 1 & 2.9523 & 1.6926 & 2.1772 \\
\hline SISO & 1 & 1 & 1.48899 & 1.4780 & 1.6936 \\
\hline
\end{tabular}

TABLE II: Overall achievable and actual rates gains per cell, w.r.t SISO networks, for the different MIMO setups, in an interference-limited scenario for $M=4,16$-QAM modulation scheme.

\section{B. Diversity-Multiplexing Tradeoffs \& Design Guidelines}

Using similar parameters as Fig. 1, the results in Table II compare the performance of the considered MIMO configurations in terms of error probability, outage probability, ergodic rate, and throughput, ${ }^{9}$ to quantify the achievable gains w.r.t the SISO configuration. The results in both Fig. 1 and Table II clearly show the diversity-multiplexing tradeoff in cellular networks. The results show the outage probability improvement due to diversity, in which the OSTBC achieves the highest outage probability reduction. This is because OSTBC provides both transmit and receive diversity while MISO and SIMO provide either transmit or receive diversity. Note that despite that MISO and SIMO have the same performance, the

\footnotetext{
${ }^{9}$ The throughput is defined as the number of successfully transmitted bits pcu and is given by $\log _{2}(M)(1-\mathrm{ASEP})$.
}

SIMO is preferred because it relies on the receive CSI which is easier to obtain than the transmit CSI. The results also show the negative impact of multiplexing on the per-stream ASEP and outage probability in ZF-Rx and MU-MIMO schemes. However, multiplexing several streams per BS improves the overall ergodic rate and per-cell throughput as shown in Table II.

The results in Fig. 1 and Table II show the diversitymultiplexing tradeoffs that can be achieved for a $2 \times 2 \mathrm{MIMO}$ setting. However, as $N_{t}$ and $N_{r}$ grow, several diversity and multiplexing tradeoffs are no longer straightforward to compare. Hence, it is beneficial to have a unified methodology to select the appropriate diversity, multiplexing, and number of antennas to meet a certain design objective. From Proposition 1 and the subsequent results we noticed two important insights: (i)The performances of MIMO schemes differ according to their relative $m_{o}$ and $m_{i}$ values. In other words, MIMO configurations with equal $\frac{m_{o}}{m_{i}}$ ratio have approximately equivalent per-stream performance as shown in Fig. 3(a). Moreover, such equivalence in performance can be further verified by the numerical results of [16] where similar ASEP performance among different MIMO schemes having the same fading parameters has been reported. (ii) Multiplexing more data streams increases $m_{i}$ and does not affect $m_{o}$. On the other hand, diversity increases $m_{o}$ and does not affect $m_{i}$. In other words, $m_{o}$ represents the diversity gain and $m_{i}$ represents the number of independently multiplexed data streams per BS (i.e., multiplexing gain). Based on the aforementioned insights, we plot the unified MIMO outage probability and ASEP performance results in Fig. 3. The Figs. 3(b) and 3(c) show the ASEP and outage probability for a varying ratio of $\frac{m_{o}}{m_{i}}$ which can be used for all considered MIMO schemes. Conversely, Fig. 3 presents a unified design methodology for MIMO cellular networks as shown in Fig. 4. Such unified design provides reliable guidelines for network designers and defines the different flavors of the considered MIMO configurations in terms of achievable diversity and/or multiplexing gains. For instance, for an ASEP or outage probability constraint, the corresponding ratio $\frac{m_{o}}{m_{i}}$ and modulation scheme are determined. Then, the network designer can determine the MIMO technique depending on the number of data streams (or number of users) that need to be simultaneously served (i.e., determine $L$ or $\mathcal{K}$ ). Finally, the number of transmit and receive antennas for the selected MIMO scheme can be determined from Table I. Figs. 3(b) and 3(c) clearly show that incrementing the ratio $\frac{m_{o}}{m_{j}}$ enhances the diversity gain whereas decrementing it provides a higher multiplexing gain. That is, network designers are able to maintain the same perstream ASEP/outage probability by appropriately adjusting the operational parameters, namely, $N_{r}, N_{t}$ and $L$ (or $\mathcal{K}$ for SDMA). This is done by compensating $m_{i}$ with the adequate $m_{o}$ such that $\frac{m_{o}}{m_{i}}$ is kept constant. For instance, consider a network that needs to increase the number of served users $\mathcal{K}$ without compromising the reliability performance of each served user. According to Table I and Fig. 3, this is achieved by keeping $\frac{m_{o}}{m_{i}}=c$, where $c$ is a constant, which hence costs the network additional $\lceil\mathcal{K}(c+1)-1\rceil$ transmit antennas per BS.

It is worth mentioning that a design based on the ASEP is more tangible as it is sensitive to the used modulation scheme and constellation size, as opposed to the outage 


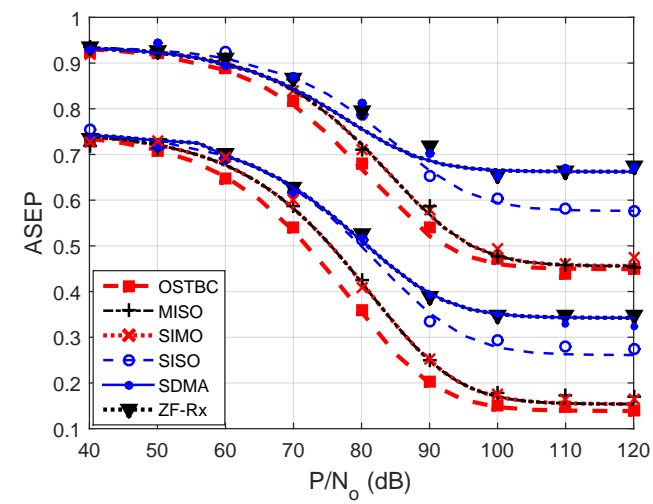

(a) ASEP for 4-QAM and 16-QAM.

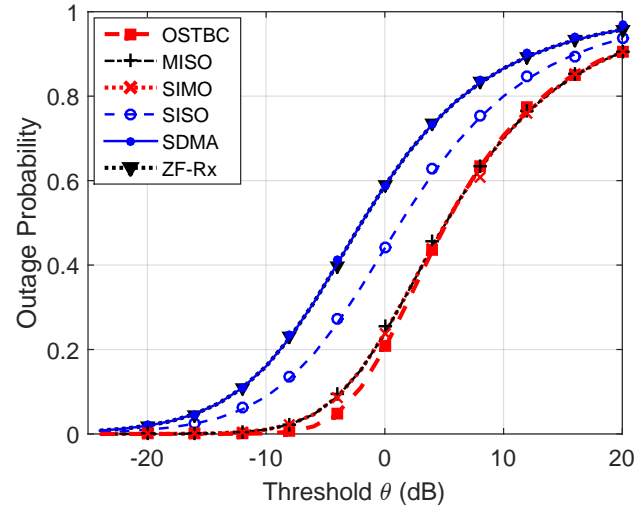

(b) Outage Probability versus SIR threshold $\theta$.

Fig. 1: ASEP and Outage probability performance validation for the different MIMO setups using the same number of antennas $N_{t}=2$ and $N_{r}=2$, at $p=1$. Lines represent the proposed analysis and markers represent Monte-Carlo simulations.

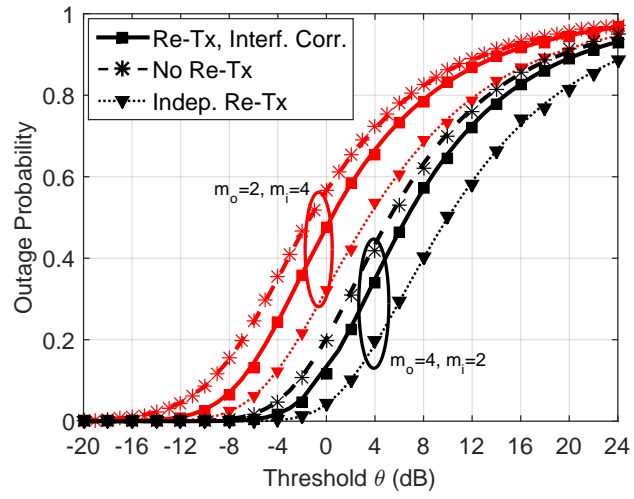

(a) The effect of interference correlation for different $m_{o}$ and $m_{i}$.

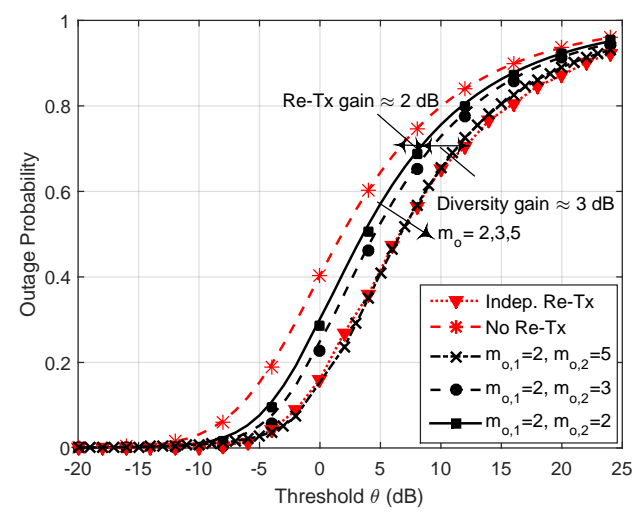

(b) Incremental diversity for the same inter-cell interference.

Fig. 2: ASEP and Outage probability performance validation. Lines represent the proposed analysis and markers represent Monte-Carlo simulations.

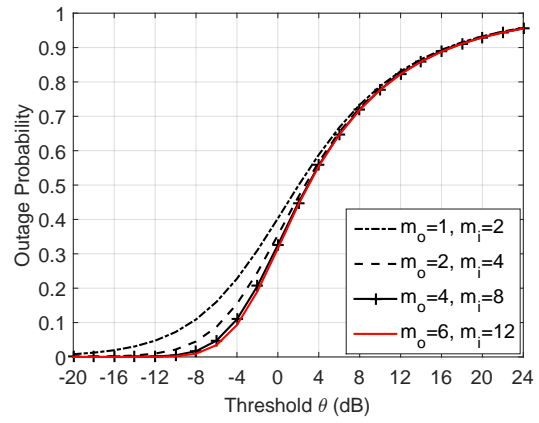

(a) Outage Probability for $\frac{m_{o}}{m_{i}}=\frac{1}{2}$.

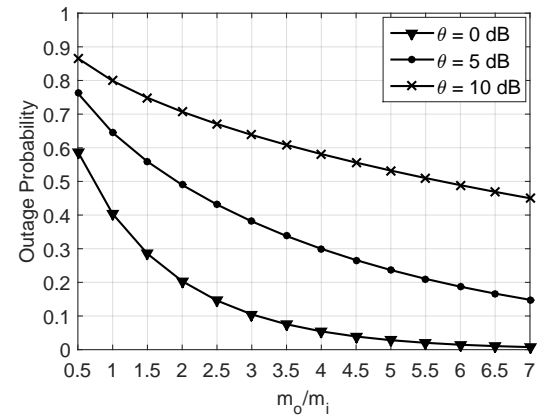

(b) Outage Probability for $\theta=0,5,10 \mathrm{~dB}$.

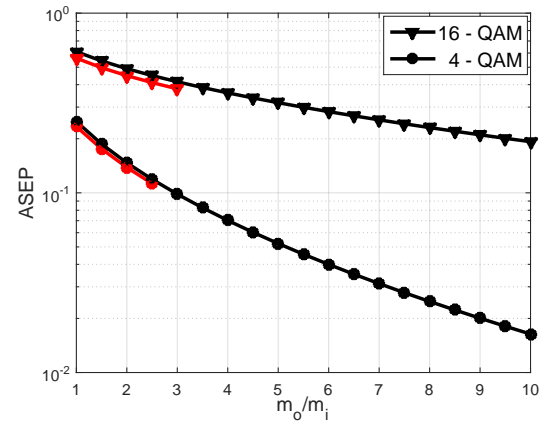

(c) ASEP for 4-QAM and 16-QAM.

Fig. 3: Unified performance versus the ratio $\frac{m_{o}}{m_{i}}$ for an arbitrary MIMO setup. 


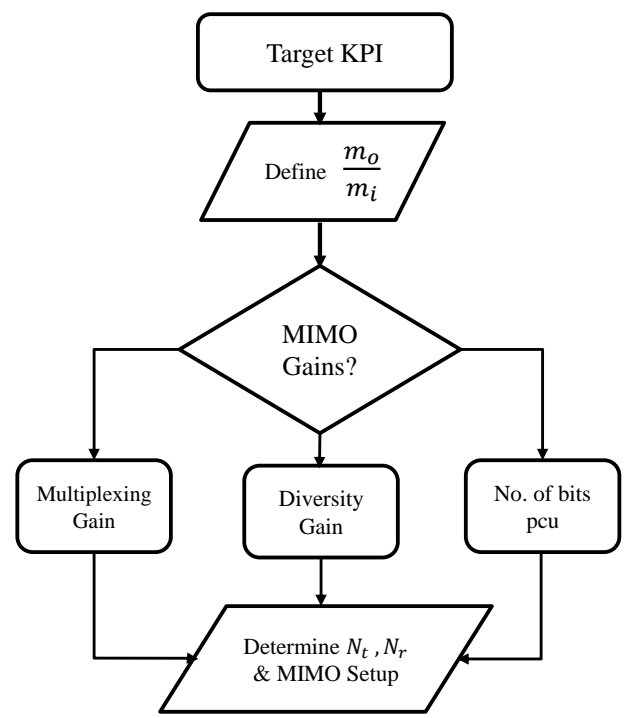

Fig. 4: Flowchart for MIMO selection in cellular networks

probability as shown in Fig. 1, and Table II. Also, note that increasing $m_{o}$ for a fixed $\frac{m_{o}}{m_{i}}$ ratio can slightly vary the outage probability due to the channel hardening effect as shown in Fig 3(a). However, such variation is shown to be negligible for $m_{o}>2$. In fact, by direct inspection of eq. (18), it is clear that as $m_{o}$ increases, the value of the summation also increases and therefore, the overall outage performance decreases, since the increase in $m_{o}$ is interpreted as an enhancement in the desired signal. Nevertheless, if the improvement in the desired signal, i.e., $m_{o}$, is compensated by an equivalent increase in the interfering signals, i.e., $m_{i}$, the performance eventually saturates and no better performance can be achieved as long as the ratio $\frac{m_{o}}{m_{i}}$ is kept constant. Another noteworthy observation is that the second term in (10), which corresponds to the $\operatorname{erfc}^{2}(\cdot)$ term in (5), requires threefold nested integrals that involve hypergeometric functions to evaluate the ASEP. Such integration is computationally complex to evaluate and may impose some numerical instability specially for large arguments of $m_{o}$ and $m_{i}$. In order to overcome such complexity and numerical instability, we invoke Jensen's inequality to the $\operatorname{erfc}^{2}(\cdot)$ term in (5). Hence, the ASEP function becomes $\operatorname{ASEP}(\Upsilon) \geq$ $w_{1} \mathbb{E}[\operatorname{erfc}(\sqrt{\beta \Upsilon})]+w_{2} \mathbb{E}[\operatorname{erfc}(\sqrt{\beta \Upsilon})]^{2}$, which reduces one integral from the second term of (10). Using Jensen's inequality yields a stable and accurate approximation compared to (10) as shown in Figure 3(c), where the red curves represent the numerically unstable ASEP performance as arguments grow, while the black curves represent the Jensen's inequality tight upper bounds.

\section{CONCLUSION}

This paper provides a unified tractable framework for studying symbol error probability, outage probability, ergodic rate, and throughput for downlink cellular networks with different MIMO configurations. The developed model also captures the effect of temporal interference correlation on the outage probability after signal retransmission. The unified analysis is achieved by Gaussian signaling approximation along with an equivalent SISO-SINR representation for the considered MIMO schemes. The accuracy of the proposed model is verified against Monte-Carlo simulations. To this end, we shed lights on the diversity loss due to temporal interference correlation and discuss the diversity-multiplexing tradeoff imposed by MIMO configurations. Finally, we propose a unified design methodology to choose the appropriate diversity, multiplexing, and number of antennas to meet a certain design objective.

\section{APPENDIX}

\section{A: Proof of THEOREM 1}

The ASEP expression in (10) is obtained by taking the expectation over $\Upsilon$ and then using expressions from [49, eq. (11), (21)] as has been detailed in [29].

For the outage probability, conditioned on $r_{o}$,

$$
\begin{aligned}
\mathcal{O}\left(r_{o}, \theta\right) & =\mathbb{E}\left[\mathbb{P}\left(g_{o}<\frac{\theta \mathcal{I}}{P r_{o}^{-\eta}}\right)\right] \\
& \stackrel{(c)}{=} \mathbb{E}\left[1-\sum_{j=0}^{m_{o}-1} \frac{1}{j !}\left(\frac{\theta \mathcal{I}}{P r_{o}^{-\eta}}\right)^{j} \exp \left\{\frac{-\theta \mathcal{I}}{P r_{o}^{-\eta}}\right\}\right],
\end{aligned}
$$

where $(c)$ follows from the CDF of the gamma distribution, and then (11) is obtained from the rules of differentiation of the LT, together with averaging over the PDF of $r_{o}$.

Ergodic rate expression in (12) follows from [50, Lemma 1], and by exploiting the independence between the useful and interfering signals, as well as incorporating the $\mathrm{CDF}$ of the gamma random variable.

\section{B: PROOF OF LEMMA 2}

Let $\tilde{\Psi}_{1}^{o} \subset \tilde{\Psi}^{o}$ and $\tilde{\Psi}_{2}^{o} \subset \tilde{\Psi}^{o}$ be the sets of interfering BSs in the first and second time slots of transmissions, respectively. Exploiting the independent transmission assumption per time slot, $\tilde{\Psi}_{1}^{o}$ and $\tilde{\Psi}_{2}^{o}$ can be decomposed into the three independent PPPs $\left\{\tilde{\Psi}_{1}^{o} \backslash \tilde{\Psi}_{2}^{o}\right\},\left\{\tilde{\Psi}_{2}^{o} \backslash \tilde{\Psi}_{1}^{o}\right\}$, and $\left\{\tilde{\Psi}_{2}^{o} \cap \tilde{\Psi}_{1}^{o}\right\}$ with intensities $p(1-p) \lambda_{B},(1-p) p \lambda_{B}$, and $p^{2} \lambda_{B}$, respectively. Substituting $p \lambda_{B}=\lambda$, the joint LT of the two random variables $\mathcal{I}_{1}$, and $\mathcal{I}_{2}$ is given as shown in (19), where $(d)$ is obtained from the PGFL and exploiting the independence between the PPPs $\left\{\tilde{\Psi}_{1}^{o} \backslash \tilde{\Psi}_{2}^{o}\right\},\left\{\tilde{\Psi}_{2}^{o} \backslash \tilde{\Psi}_{1}^{o}\right\}$, and $\left\{\Psi_{2}^{o} \cap \tilde{\Psi}_{1}^{o}\right\}$ [35], and (e) follows from the LT of the two independent gamma distributed random variables $\tilde{g}_{i}^{(1)}$ and $\tilde{g}_{i}^{(2)}$. Solving the integral completes the proof.

\section{C: Proof of Theorem 2}

The joint complementary cumulative distribution function (CCDF) of $\bar{\Upsilon}_{1}$ and $\bar{\Upsilon}_{2}$ is given by

$$
\begin{gathered}
\mathbb{P}\left(\bar{\Upsilon}_{1}>\theta, \bar{\Upsilon}_{2}>\theta\right)=\mathbb{E}\left[\mathbb{P}\left(\tilde{g}_{o}^{(1)}>\frac{\theta \mathcal{I}_{1}}{P r_{o}^{-\eta}}, \quad \tilde{g}_{o}^{(2)}>\frac{\theta \mathcal{I}_{2}}{P r_{o}^{-\eta}}\right)\right] \\
\stackrel{(i)}{=} \mathbb{E}\left[\sum_{j_{1}=0}^{m_{o, 1}-1} \sum_{j_{2}=0}^{m_{o, 2}-1} \frac{1}{j_{1} ! j_{2} !}\left(\frac{-\theta}{P r_{o}^{-\eta}}\right)^{j_{1}+j_{2}}\left(\mathcal{I}_{1}^{j_{1}} \mathcal{I}_{2}^{j_{2}}\right)\right. \\
\left.\stackrel{(i i)}{=} \mathbb{E}\left[\sum_{j_{1}=0} \sum_{j_{2}=0}^{m_{o}, 1-1} \frac{-\theta\left(\mathcal{I}_{1}+\mathcal{I}_{2}\right)}{P r_{o}^{-\eta}}\right\}\right] \\
\left.\times\left.\frac{\partial^{\left(j_{1}+j_{2}\right)}}{\partial z_{1}^{j_{1}} \partial z_{2}^{j_{2}}} \mathcal{L}_{\mathcal{I}_{1}, \mathcal{I}_{2} \mid r_{o}}\left(z_{1}, z_{2}\right)\right|_{z_{1}=z_{2}=\frac{\theta}{P r_{o}^{-\eta}}}\right]
\end{gathered}
$$




$$
\begin{aligned}
& \mathcal{L}_{\mathcal{I}_{1}, \mathcal{I}_{2} \mid r_{o}}\left(z_{1}, z_{2}\right)=\mathbb{E}\left[\exp \left\{-\sum_{r_{i} \in \tilde{\Psi}_{2}^{o} \cap \tilde{\Psi}_{1}^{o}} \operatorname{Pr}_{i}^{-\eta}\left(z_{1} \tilde{g}_{i}^{(1)}+z_{2} \tilde{g}_{i}^{(2)}\right)-z_{1} \sum_{r_{i} \in \tilde{\Psi}_{1}^{o} \backslash \tilde{\Psi}_{2}^{o}} \operatorname{Pr}_{i}^{-\eta} \tilde{g}_{i}^{(1)}-z_{2} \sum_{r_{i} \in \tilde{\Psi}_{2}^{o} \backslash \tilde{\Psi}_{1}^{o}} \operatorname{Pr}_{i}^{-\eta} \tilde{g}_{i}^{(2)}\right\}\right] \\
& \stackrel{(d)}{=} \exp \left\{-2 \pi p \lambda \int_{r_{o}}^{\infty} \mathbb{E}_{\tilde{g}_{i}^{(1)}, \tilde{g}_{i}^{(2)}}\left[1-e^{-P x^{-\eta}\left(z_{1} \tilde{g}_{i}^{(1)}+z_{2} \tilde{g}_{i}^{(2)}\right)}\right] x \mathrm{~d} x-2 \pi(1-p) \lambda \int_{r_{o}}^{\infty} \mathbb{E}_{\tilde{g}_{i}^{(1)}}\left[1-e^{-P x^{-\eta} z_{1} \tilde{g}_{i}^{(1)}}\right] x \mathrm{~d} x\right. \\
& \left.-2 \pi(1-p) \lambda \int_{r_{o}}^{\infty} \mathbb{E}_{\tilde{g}_{i}^{(2)}}\left[1-e^{-P x^{-\eta} z_{2} \tilde{g}_{i}^{(2)}}\right] x \mathrm{~d} x\right\} \\
& \stackrel{(e)}{=} \exp \left\{-2 \pi \lambda \int_{r_{o}}^{\infty}\left(p\left[1-\frac{1}{\left(1+P x^{-\eta} z_{1}\right)^{m_{i, 1}}} \frac{1}{\left(1+P x^{-\eta} z_{2}\right)^{m_{i, 2}}}\right]+(1-p)\left[1-\frac{1}{\left(1+P x^{-\eta} z_{1}\right)^{m_{i, 1}}}\right]\right.\right. \\
& \left.\left.+(1-p)\left[1-\frac{1}{\left(1+P x^{-\eta} z_{2}\right)^{m_{i, 2}}}\right]\right) x \mathrm{~d} x\right\}
\end{aligned}
$$

such that $(i)$ follows from the independence of $\tilde{g}_{o}^{(1)}$ and $\tilde{g}_{o}^{(2)}$ along with the CCDF of their Gamma distributions. ( $i$ i $)$ is obtained by utilizing the LT identity $t_{1}^{a} t_{2}^{b} f\left(t_{1}, t_{2}\right) \Leftrightarrow$ $\frac{\partial^{(a+b)}}{\partial z_{1}^{a} \partial z_{2}^{b}} \mathcal{L}_{t_{1}, t_{2}}\left(z_{1}, z_{2}\right)$, which can be proved as follows. First, we write the joint LT of two variables $t_{1}$ and $t_{2}$ as

$$
\mathcal{L}_{t_{1}, t_{2}}\left(z_{1}, z_{2}\right)=\int_{0}^{\infty} \int_{0}^{\infty} f\left(t_{1}, t_{2}\right) e^{-z_{1} t_{1}} e^{-z_{2} t_{2}} \mathrm{~d} t_{1} \mathrm{~d} t_{2},
$$

then,

$$
\begin{aligned}
& \frac{\partial^{j_{1}+j_{2}} \mathcal{L}_{t_{1}, t_{2}}\left(z_{1}, z_{2}\right)}{\partial z_{1}^{j_{1}} \partial z_{2}^{j_{2}}} \\
& =\frac{\partial^{j_{1}+j_{2}}}{\partial z_{1}^{j_{1}} \partial z_{2}^{j_{2}}} \int_{0}^{\infty} \int_{0}^{\infty} f\left(t_{1}, t_{2}\right) e^{-z_{1} t_{1}} e^{-z_{2} t_{2}} \mathrm{~d} t_{1} \mathrm{~d} t_{2} \\
& =\int_{0}^{\infty} \int_{0}^{\infty}(-1)^{j_{1}+j_{2}}\left(t_{1}\right)^{j_{1}}\left(t_{2}\right)^{j_{2}} f\left(t_{1}, t_{2}\right) e^{-z_{1} t_{1}} e^{-z_{2} t_{2}} \mathrm{~d} t_{1} \mathrm{~d} t_{2},
\end{aligned}
$$

where the second equality follows by Leibniz rule and applying the rules of partial differentiation, which proves the identity.

\section{D: Equivalent SiSO Model Proofs}

Proof of Lemma 3: In SIMO transmission, by applying MRC at the receiver side, for $\overline{\mathbf{w}}_{o}^{T}=\mathbf{h}_{o}^{H}$, then the postprocessed signal is written as

$$
\begin{aligned}
\tilde{y} & =\overline{\mathbf{w}}_{o}^{T} \mathbf{y} \\
& =\sqrt{P} r_{o}^{-\frac{\eta}{2}}\left\|\mathbf{h}_{o}\right\|^{2} s_{o}+\sum_{r_{i} \in \tilde{\Psi}^{o}} \sqrt{P} r_{i}^{-\frac{\eta}{2}} \mathbf{h}_{o}^{H} \mathbf{h}_{i} \tilde{s}_{i}+\mathbf{h}_{o}^{H} \mathbf{n} .
\end{aligned}
$$

We start with computing the effective noise variance since a post-processor is applied. The noise power is expressed as

$$
\operatorname{Var}_{\mathbf{n}}\left[\mathbf{h}_{o}^{H} \mathbf{n}\right]=\mathcal{N}_{o}\left\|\mathbf{h}_{o}\right\|^{2} .
$$

Therefore, the random variable $\epsilon=\left\|\mathbf{h}_{o}\right\|^{2}$, is used to normalize the resultant interference power. The effective interference variance conditioned on the network geometry and the intended channel gains w.r.t $\tilde{s}_{i}$ is given by

$$
\mathcal{I}=\frac{1}{\epsilon} \operatorname{Var}_{\tilde{s}_{i}}\left[\sum_{r_{i} \in \tilde{\Psi}^{o}} \sqrt{P} r_{i}^{-\frac{\eta}{2}} \mathbf{h}_{o}^{H} \mathbf{h}_{i} \tilde{s}_{i}\right]=\sum_{r_{i} \in \tilde{\Psi}^{o}} \operatorname{Pr}_{i}^{-\eta} \frac{\left|\mathbf{h}_{o}^{H} \mathbf{h}_{i}\right|^{2}}{\left\|\mathbf{h}_{o}\right\|^{2}} .
$$

By inspection of the interference variance, it is clear that $\{\breve{\mathbf{H}}\}=\left\{\mathbf{H}_{o}\right\}$. Also, we notice that there exists only one coefficient $a_{l}^{(i)}=\frac{\mathbf{h}_{o}^{H} \mathbf{h}_{i}}{\left\|\mathbf{h}_{o}\right\|}$. Recall that the number of independent coefficients $a_{l, k}^{(i)}$ depends on the number of independent transmitted streams, which is equal to one in the SIMO case.
Accordingly, $\tilde{g}_{i}=\left|a_{l}^{(i)}\right|^{2} \sim \operatorname{Gamma}\left(m_{i}, 1\right)$, with $m_{i}=1$. Similarly, conditioned on the intended and interfering channel gains, the received signal power, w.r.t the transmitted signal, can be shown to be

$$
\mathcal{S}=\frac{1}{\epsilon} \operatorname{Var}_{s_{o}}\left[\sqrt{P} r_{o}^{-\frac{\eta}{2}}\left\|\mathbf{h}_{o}\right\|^{2} s_{o}\right]=\operatorname{Pr}_{o}^{-\eta}\left\|\mathbf{h}_{o}\right\|^{2} .
$$

Therefore, $\tilde{g}_{o}=\left\|\mathbf{h}_{o}\right\|^{2} \sim \operatorname{Gamma}\left(m_{o}, 1\right)$ where $m_{o}=N_{r}$.

Proof of Lemma 4: Employing OSTBC, the received vector at a typical user at time instant $\tau, N_{t} \leq \tau \leq T$, is given by

$$
\mathbf{y}(\tau)=\sqrt{P} r_{o}^{-\frac{\eta}{2}} \mathbf{H}_{o} \mathbf{s}+\sum_{r_{i} \in \tilde{\Psi}^{o}} \sqrt{P} r_{i}^{-\frac{\eta}{2}} \mathbf{H}_{i} \tilde{\mathbf{s}}_{i}+\mathbf{n}(\tau) .
$$

Let $\mathcal{Y}$ be the stacked vector of received symbols over $T$ intervals, and let $L=N_{t}$, such that,

$$
\mathcal{Y}=\sqrt{P} r_{o}^{-\frac{\eta}{2}} \mathbf{H}_{\text {eff }} \mathbf{s}+\mathbf{i}_{\text {agg }}+\mathbf{n} .
$$

where $\mathcal{Y} \in \mathbb{C}^{T \cdot N_{r} \times 1}$, and $\mathbf{i}_{\text {agg }}$ is the concatinated aggregate interference $T \cdot N_{r} \times 1$ vector. The effective channel matrix $\mathbf{H}_{\text {eff }} \in \mathbb{C}^{T \cdot N_{r} \times N_{t}}$ is expressed as a linear combination of the set of dispersion matrices $\mathcal{A}$ and $\mathcal{B}$ chosen according to the adopted orthogonal space-time code as follows [3], [51],

$$
\mathbf{H}_{\mathrm{eff}}=\sum_{j=1}^{N_{r}} \sum_{q=1}^{N_{t}} \alpha_{j q} \mathcal{A}_{j q}+\jmath \beta_{j q} \mathcal{B}_{j q}
$$

where $h_{j q}=\alpha_{j q}+\jmath \beta_{j q}$. Moreover, $\mathbf{H}_{\mathrm{eff}}^{H} \mathbf{H}_{\mathrm{eff}}=\left\|\mathbf{H}_{o}\right\|_{\mathrm{F}}^{2} \mathbf{I}$ where $\left\|\mathbf{H}_{o}\right\|_{\mathrm{F}}^{2}=\sum_{j=1}^{N_{r}} \sum_{q=1}^{N_{t}}\left|h_{j q}\right|^{2}$ is the squared Frobenius norm of the intended channel matrix. Hence, $\left\|\mathbf{H}_{o}\right\|_{\mathrm{F}}^{2} \sim$ $\frac{1}{2} \chi^{2}\left(2 N_{s} N_{r}\right)$ [1]. Moreover, the aggregate interfering signals are expressed as

$$
\mathbf{i}_{\text {agg }}=\sum_{r_{i} \in \tilde{\Psi}^{o}} \sqrt{P} r_{i}^{-\frac{\eta}{2}} \mathbf{H}_{i, \mathrm{eff}} \tilde{\mathbf{s}}_{i},
$$

such that $\mathbf{H}_{i, \text { eff }}$ is defined similar to (29). For detection, we equalize the effective channel matrix at the receiver side by $\mathbf{W}_{o}$. Hence, the received vector $\tilde{\mathcal{Y}}$ is written as

$$
\tilde{\mathcal{Y}}=\mathbf{W}_{o} \mathcal{Y}=\sqrt{P} r_{o}^{-\frac{\eta}{2}}\left\|\mathbf{H}_{o}\right\|_{\mathrm{F}} \mathbf{s}+\sum_{r_{i} \in \tilde{\Psi}^{o}} \sqrt{P} r_{i}^{-\frac{\eta}{2}} \mathbf{A}_{i} \tilde{\mathbf{s}}_{i}+\mathbf{w},
$$

such that $\mathbf{w}=\mathbf{W}_{o} \mathbf{n}$ and $\mathbf{A}_{i}=\mathbf{W}_{o} \mathbf{H}_{i, \text { eff }}$ with elements $a_{l, k}^{(i)}$ as defined in (2). Without loss of generality, we consider the detection of the $l^{t h}$ arbitrary symbol from the received vector $\tilde{\mathcal{Y}}$. Due to the adopted Gaussian signaling scheme, we lump interfernce with noise, and thus it is essential to obtain the interference variance. First, let us define $\boldsymbol{q}_{k}$ as the $k^{t h}$ column of the matrix $\mathbf{H}_{\text {eff }}$, similarly, $\boldsymbol{q}_{i, k}$ is the $k^{t h}$ column of the matrix $\mathbf{H}_{i, \text { eff. Then, the received interference variance for }}$ 
the $l^{\text {th }}$ symbol denoted as $\mathcal{I}_{l}$, computed w.r.t the interfering symbols $\widetilde{\mathbf{s}}_{i}$ can be derived as

$$
\begin{aligned}
\mathcal{I}_{l} & =\operatorname{Var}_{\mathbf{s}_{i}}\left[\sum_{r_{i} \in \tilde{\Psi}^{o}} \sum_{k=1}^{N_{s}} \sqrt{\operatorname{P}} r_{i}^{-\frac{\eta}{2}} \frac{\boldsymbol{q}_{l}^{H} \boldsymbol{q}_{i, k}}{\left\|\mathbf{H}_{o}\right\|_{\mathbf{F}}} s_{i, k}\right] \\
& =\sum_{r_{i} \in \tilde{\Psi}^{o}} \sum_{k=1}^{N_{s}} \operatorname{Pr}_{i}^{-\eta} \frac{\left|\boldsymbol{q}_{l}^{H} \boldsymbol{q}_{i, k}\right|^{2}}{\left\|\mathbf{H}_{o}\right\|_{\mathbf{F}}^{2}},
\end{aligned}
$$

where the summation is over the $N_{s}$ active antennas per transmission. Note that, conditioned on $\{\breve{\mathbf{H}}\}=\left\{\mathbf{H}_{o}\right\}, a_{l, k}^{(i)}=$ $\frac{\boldsymbol{q}_{l}^{H} \boldsymbol{q}_{i, k}}{\left\|\mathbf{H}_{o}\right\|_{\mathbf{F}}}$ is a normalized and independently weighted sum of complex Gaussian random variables, thus $a_{l, k}^{(i)} \sim \mathcal{C N}(0,1)$. Although a post-processor is applied, the noise power is maintained to be $\mathcal{N}_{o}$. Thus, $\tilde{g}_{i} \sim\left(m_{i}, \Omega_{i}\right)$ with $m_{i}=N_{s}$ and $\Omega_{i}=1$. Similarly, the received signal power is found to be

$$
\mathcal{S}=\operatorname{Var}_{\mathrm{s}}\left[\sqrt{P} r_{o}^{-\frac{\eta}{2}}\left\|\mathbf{H}_{o}\right\|_{\mathrm{F}}\right]=P r_{o}^{-\eta}\left\|\mathbf{H}_{o}\right\|_{\mathrm{F}}^{2},
$$

where $\tilde{g}_{o} \sim \operatorname{Gamma}\left(m_{o}, \Omega_{o}\right)$ with $m_{o}=N_{s} N_{r}$ and $\Omega_{o}=1$.

Proof of Lemma 5: Without loss of generality, we focus on the detection of an arbitrary symbol $l$ from the received vector $\tilde{\mathbf{y}}=\mathbf{W}_{o} \mathbf{y}$, given by

$$
\tilde{y}_{l}=\sqrt{P} r_{o}^{-\frac{\eta}{2}} s_{l}+\sum_{r_{i} \in \tilde{\Psi}^{o}} \sqrt{P} r_{i}^{-\frac{\eta}{2}} \overline{\mathbf{w}}_{o, l}^{T} \mathbf{H}_{i} \tilde{\mathbf{s}}_{i}+\overline{\mathbf{w}}_{o, l}^{T} \mathbf{n},
$$

which is similar to (2). First we need to to obtain the received noise variance since a post-processing matrix is applied and thus the noise variance is scaled. Conditioned on $\mathbf{H}_{o}$, the received noise power is defined as

$$
\begin{aligned}
\operatorname{Var}_{\mathbf{n}}\left[\overline{\mathbf{w}}_{o, l}^{T} \mathbf{n}\right] & =\overline{\mathbf{w}}_{o, l}^{T} \mathbb{E}\left[\mathbf{n n}^{H}\right] \overline{\mathbf{w}}_{o, l}^{*}=\mathcal{N}_{o}\left(\mathbf{W}_{o} \mathbf{W}_{o}^{H}\right)_{l l} \\
& =\mathcal{N}_{o}\left(\mathbf{H}_{o} \mathbf{H}_{o}^{H}\right)_{l l}^{-1} .
\end{aligned}
$$

Then, the scaling random variable is $\epsilon=\left(\mathbf{H}_{o} \mathbf{H}_{o}^{H}\right)_{l l}^{-1}$. Next, we obtain the effective interference variance from the $l^{\text {th }}$ received symbol as

$$
\begin{aligned}
\mathcal{I}_{l} & =\frac{1}{\epsilon} \operatorname{Var}_{\tilde{\mathbf{s}}_{i}}\left[\sum_{r_{i} \in \tilde{\Psi}^{o}} \sqrt{P} r_{i}^{-\frac{\eta}{2}} \overline{\mathbf{w}}_{o, l}^{T} \mathbf{H}_{i} \tilde{\mathbf{s}}_{i}\right] \\
& =\frac{1}{\epsilon} \sum_{r_{i} \in \tilde{\Psi}^{o}} \operatorname{Pr}_{i}^{-\eta}\left(\mathbf{W}_{o} \mathbf{W}_{o}^{H}\right)_{l l}\left(\mathbf{H}_{i} \mathbf{H}_{i}^{H}\right)_{l l}=\sum_{r_{i} \in \tilde{\Psi}^{o}} \operatorname{Pr}_{i}^{-\eta}\left(\mathbf{H}_{i} \mathbf{H}_{i}^{H}\right)_{l l} .
\end{aligned}
$$

The processing resulting interference channel set $\{\breve{\mathbf{H}}\}=\emptyset$. Therefore, $a_{l, k}^{(i)}=\left(\mathbf{H}_{i} \mathbf{H}_{i}^{H}\right)_{l l}$ and $\tilde{g}_{i} \sim\left(m_{i}, \Omega_{i}\right)$, with $m_{i}=N_{t}$ and $\Omega_{i}=1$. The received signal power is similarly computed as

$$
\mathcal{S}=\frac{1}{\epsilon} \operatorname{Var}_{\mathbf{s}}\left[\sqrt{P} r_{o}^{-\frac{\eta}{2}} s_{l}\right]=\frac{P r_{o}^{-\eta}}{\left(\mathbf{H}_{o} \mathbf{H}_{o}^{H}\right)_{l l}^{-1}} .
$$

Since $g_{w}=\left(\mathbf{H}_{o} \mathbf{H}_{o}^{H}\right)_{l l}^{-1} \sim$ Inv-Gamma $\left(N_{r}-N_{t}+1,1\right)$ [1]. Then, we can let $\frac{1}{g_{w}}=\tilde{g}_{o} \sim \operatorname{Gamma}\left(m_{o}, \Omega_{o}\right)$, where $m_{o}=$ $N_{r}-N_{t}+1$ and $\Omega_{o}=1$.
Proof of Lemma 6: In a multi-user MIMO setting, we introduce a slight abuse of notation for the intended and interfering channel matrices such that they are of dimensions $\mathcal{K} \times N_{t}$. The received interference power at user $l$ where $1 \leq l \leq \mathcal{K}$, averaged over the interfering symbols $\tilde{s}_{\boldsymbol{i}}$ is given by

$$
\mathcal{I}_{l}=\operatorname{Var}_{\tilde{s}_{\boldsymbol{i}}}\left[\sum_{r_{i} \in \tilde{\Psi}^{o}} \sqrt{P} r_{i}^{-\frac{\eta}{2}} \mathbf{h}_{i, l} \mathbf{V}_{i} \tilde{\boldsymbol{s}}_{\boldsymbol{i}}\right]=\sum_{r_{i} \in \tilde{\Psi}^{o}} \operatorname{Pr}_{i}^{-\eta}\left\|\mathbf{h}_{i, l} \mathbf{V}_{i}\right\|^{2},
$$

where $\mathbf{h}_{i, l}$ is the $l^{\text {th }}$ row of $\mathbf{H}_{i}$ and $\{\breve{\mathbf{H}}\}=\left\{\mathbf{V}_{i}\right\}$. Also, $\left\|\mathbf{h}_{i, l} \mathbf{V}_{i}\right\|^{2}=\sum_{l=1}^{\mathcal{K}}\left|a_{l, k}^{(i)}\right|^{2}$. However, the column vectors of $\mathbf{V}_{i}$ are not independent. Therefore, conditioned on $\mathbf{v}_{i, l}$, the linear combination $\sum_{l=1}^{\mathcal{K}}\left|a_{l, k}^{(i)}\right|^{2}$ does not follow a Gamma distribution. Nevertheless, for tractability we approximate this summation by a Gamma distribution. Thus, $\tilde{g}_{i} \sim \operatorname{Gamma}\left(m_{i}, \Omega_{i}\right)$, where $m_{i}=\mathcal{K}$ and $\Omega_{i}=1$ by assuming such independence. This renders the aggregate interference power distribution at user $l$ an approximation. Similarly, the useful signal power at user $l$ is straightforward to be obtained, after appropriate diagonalization, as $\mathcal{S}=\frac{1}{\left\|\mathbf{v}_{l}\right\|^{2}} \sim \operatorname{Gamma}\left(m_{o}, \Omega_{o}\right)$, with $m_{o}=N_{t}-\mathcal{K}+1$ and $\Omega_{o}=1$ [2]. This can also be interpreted as having the precoding matrix nulling out $\mathcal{K}-1$ directions out of the $N_{t}$ subspace at the transmitter side.

Proof of Lemma 7: Since, there are $N_{t}$ distinct multiplexed symbols to be transmitted, we will study the pairwise error probability (PEP) of two distinct transmitted codewords, denoted as $\mathcal{P}=\mathbb{P}\left\{\tilde{\mathbf{s}}=\mathbf{s}_{1} \mid \mathbf{s}_{o}\right\}$. For ease of notaion, let $\delta=\sqrt{P} r_{o}^{-\frac{\eta}{2}}$. Therefore,

$$
\left\|\tilde{\mathbf{y}}-\delta \mathbf{H}_{o} \mathbf{s}_{o}\right\|^{2} \underset{\mathbf{s}_{o}}{\stackrel{\mathbf{s}_{1}}{\gtrless}}\left\|\tilde{\mathbf{y}}-\delta \mathbf{H}_{o} \mathbf{s}_{1}\right\|^{2} .
$$

Using some mathematical manipulations, and assuming $\mathbf{s}_{o}$ was the actual transmitted symbols, it can be shown that

$$
\mathcal{P}(\mathbf{e})=\mathbb{P}\left\{\left[\mathbf{I}_{\text {agg }}^{H}+\mathbf{n}^{H}\right] \mathbf{H}_{o} \mathbf{e}+\mathbf{e}^{H} \mathbf{H}_{o}^{H}\left[\mathbf{I}_{\mathrm{agg}}+\mathbf{n}\right]>\delta \mathbf{e}^{H} \mathbf{H}_{o}^{H} \mathbf{H}_{o} \mathbf{e}\right\},
$$

where $\mathbf{I}_{\mathrm{agg}}=\sum_{r_{i} \in \tilde{\Psi}^{o}} I_{i}$. Conditioned on the channel matrices $\mathbf{H}_{o}$ and $\mathbf{H}_{i}$, and considering the Gaussian signaling approximation, the L.H.S of the above inequality represents the interference-plus-noise power and is a Gaussian random variable, denoted as $\mathcal{V}$ with zero-mean and variance $\sigma_{\mathcal{V}}^{2}$, thus, $\mathcal{P}(\mathbf{e})=\frac{1}{2} \operatorname{erfc}\left(\frac{\delta \mathbf{e}^{H} \mathbf{H}_{o}^{H} \mathbf{H}_{o} \mathbf{e}}{\sqrt{2 \sigma_{\mathcal{V}}^{2}}}\right)$, where the variance $\sigma_{\mathcal{V}}^{2}$ is given by

$\sigma_{\mathcal{V}}^{2}=2\left[\mathbf{e}^{H} \mathbf{H}_{o}^{H} \mathbf{H}_{o} \mathbf{e}\right]\left(\mathcal{N}_{o}+\sum_{r_{i} \in \tilde{\Psi}^{o}} \operatorname{Pr}_{i}^{-\eta} \sum_{k=1}^{N_{t}} \frac{\left|\left(\mathbf{H}_{o} \mathbf{e}\right)^{H} \mathbf{h}_{i, k}\right|^{2}}{\left\|\mathbf{H}_{o} \mathbf{e}\right\|_{2}^{2}}\right)$.

By following the same convention used in this paper, it is clear that the interference power is represented as

$$
\mathcal{I}_{l}=\sum_{r_{i} \in \tilde{\Psi}^{o}} \operatorname{Pr}_{i}^{-\eta} \sum_{k=1}^{N_{t}} \frac{\left|\left(\mathbf{H}_{o} \mathbf{e}\right)^{H} \mathbf{h}_{i, k}\right|^{2}}{\left\|\mathbf{H}_{o} \mathbf{e}\right\|^{2}}
$$

and thus we see that $\{\breve{\mathbf{H}}\}=\left\{\mathbf{H}_{o}\right\}$ and $a_{l, k}^{(i)}=\frac{\left(\mathbf{H}_{o} \mathbf{e}\right)^{H} \mathbf{h}_{i, k}}{\left\|\mathbf{H}_{o} \mathbf{e}\right\|}$. Conditioned on $\mathbf{H}_{o}, \tilde{g}_{i} \sim \operatorname{Gamma}\left(N_{t}, 1\right)$. Furthermore, let 
$\mathcal{S}=\mathbf{e}^{H} \mathbf{H}_{o}^{H} \mathbf{H}_{o} \mathbf{e} \stackrel{d}{=}\|\mathbf{e}\|^{2}\left(\mathbf{H}_{o}^{H} \mathbf{H}_{o}\right)_{l l}$, hence it is starightforward to see that $\left(\mathbf{H}_{o}^{H} \mathbf{H}_{o}\right)_{l l}$ has a $\chi^{2}\left(N_{r}\right)$ distribution. Thus, $\tilde{g}_{o}=\left(\mathbf{H}_{o}^{H} \mathbf{H}_{o}\right)_{l l} \sim \operatorname{Gamma}\left(m_{o}, \Omega_{o}\right)$, with $m_{o}=N_{r}$ and $\Omega_{o}=1$. Then, the conditional pairwise error probability is expressed by

$$
\mathcal{P}(\mathbf{e})=\frac{1}{2} \operatorname{erfc}\left(\sqrt{\frac{P r_{o}^{-\eta}\|\mathbf{e}\|^{2} \tilde{g}_{o}}{4\left(\mathcal{N}_{o}+\sum_{r_{i} \in \tilde{\Psi}^{o}} \operatorname{Pr}_{i}^{-\eta} \tilde{g}_{i}\right)}}\right) .
$$

\section{REFERENCES}

[1] R. Nabar A. Paulraj and D. Gore., Introduction to Space-Time Wireless Communications, Cambridge University Press, Cambridge, UK, 2003.

[2] C. B. Papadias H. Huang and S. Venkatesan, MIMO Communication for Cellular Networks, Springer, 2012.

[3] E. Larsson and P. Stoica, Space-Time Block Coding for Wireless Communications, Cambridge University Press, Cambridge, UK, 2003.

[4] R. Tanbourgi, H. S. Dhillon, and F. K. Jondral, "Analysis of joint transmit-receive diversity in downlink MIMO heterogeneous cellular networks," IEEE Trans. Wireless Commun., accepted 2015.

[5] R. Tanbourgi and F. K. Jondral, "Downlink MIMO diversity with maximal-ratio combining in heterogeneous cellular networks," in IEEE International Conference on Communications (ICC), June 2015, pp. 1831-1837.

[6] T. M. Nguyen, Y. Jeong, T.Q.S. Quek, W. P. Tay, and H. Shin, "Interference alignment in a Poisson field of MIMO femtocells," IEEE Trans. Wireless Commun., vol. 12, no. 6, pp. 2633-2645, Jun. 2013.

[7] A. Adhikary, H. S. Dhillon, and G. Caire, "Massive-MIMO meets HetNet: Interference coordination through spatial blanking," IEEE J. Sel. Areas Commun., accepted 2015.

[8] N. Lee, D. M.-Jimenez, A. Lozano, and R.W. Heath, "Spectral efficiency of dynamic coordinated beamforming: A stochastic geometry approach," IEEE Trans. Wireless Commun., vol. 14, no. 1, pp. 230241, Jan. 2015.

[9] A.H. Sakr and E. Hossain, "Location-aware cross-tier coordinated multipoint transmission in two-tier cellular networks," IEEE Trans. Wireless Commun., vol. 13, no. 11, pp. 6311-6325, Nov. 2014.

[10] G. Nigam, P. Minero, and M. Haenggi, "Coordinated multipoint joint transmission in heterogeneous networks," IEEE Trans. Commun., vol. 62, no. 11, pp. 4134-4146, Nov. 2014.

[11] X. Zhang and M. Haenggi, "A stochastic geometry analysis of intercell interference coordination and intra-cell diversity," IEEE Trans. Wireless Commun., vol. 13, no. 12, pp. 6655-6669, Dec. 2014.

[12] G. Nigam, P. Minero, and M. Haenggi, "Spatiotemporal cooperation in heterogeneous cellular networks," IEEE J. Sel. Areas Commun., vol. 33, no. 6, pp. 1253-1265, June 2015.

[13] R. Tanbourgi, S. Singh, J. G. Andrews, and F. K. Jondral, "A tractable model for noncoherent joint-transmission base station cooperation," vol. 13, no. 9, pp. 4959-4973, Sept 2014.

[14] H. S. Dhillon, M. Kountouris, and J. G. Andrews, "Downlink MIMO HetNets: Modeling, ordering results and performance analysis," IEEE Trans. Wireless Commun., vol. 12, no. 10, pp. 5208-5222, Oct. 2013.

[15] A. K. Gupta, H. S. Dhillon, S. Vishwanath, and J. G. Andrews, "Downlink multi-antenna heterogeneous cellular network with load balancing," IEEE Trans. Commun., vol. 62, no. 11, pp. 4052-4067, Nov. 2014.

[16] M. Di Renzo and W. Lu, "Stochastic geometry modeling and performance evaluation of MIMO cellular networks using the equivalent-indistribution (EiD)-based approach," IEEE Trans. Commun., vol. 63, no. 3, pp. 977-996, Mar. 2015.

[17] M. Di Renzo and Peng Guan, "A mathematical framework to the computation of the error probability of downlink mimo cellular networks by using stochastic geometry," IEEE Trans. Commun., vol. 62, no. 8, pp. 2860-2879, Aug. 2014.

[18] S. Govindasamy, D. W. Bliss, and D. H. Staelin, "Asymptotic spectral efficiency of the uplink in spatially distributed wireless networks with multi-antenna base stations," IEEE Trans. Commun., vol. 61, no. 7, pp. 100-112, July 2013
[19] M. Di Renzo and P. Guan, "Stochastic geometry modeling and systemlevel analysis of uplink heterogeneous cellular networks with multiantenna base stations," IEEE Transactions on Communications, vol. 64, no. 6, pp. 2453-2476, June 2016.

[20] C. Li, J. Zhang, J. G. Andrews, and K. B. Letaief, "Success probability and area spectral efficiency in multiuser MIMO hetnets," IEEE Transactions on Communications, vol. 64, no. 4, pp. 1544-1556, April 2016.

[21] J. G. Andrews, F. Baccelli, and R. K. Ganti, "A tractable approach to coverage and rate in cellular networks," IEEE Trans. Commun., vol. 59, no. 11, pp. 3122-3134, Nov. 2011.

[22] A. Guo and M. Haenggi, "Spatial stochastic models and metrics for the structure of base stations in cellular networks," IEEE Trans. Wireless Commun., vol. 12, no. 11, pp. 5800-5812, Nov. 2013.

[23] H. ElSawy, E. Hossain, and M. Haenggi, "Stochastic geometry for modeling, analysis, and design of multi-tier and cognitive cellular wireless networks: A survey," IEEE Commun. Surveys Tuts., vol. 15, no. 3, pp. 996-1019, 2013.

[24] Hesham ElSawy, Ahmed Kamal Sultan-Salem, Mohamed-Slim Alouini, and Moe Z. Win, "Modeling and analysis of cellular networks using stochastic geometry: A tutorial," CoRR, vol. abs/1604.03689, 2016.

[25] Marco Di Renzo, Wei Lu, and Peng Guan, "The intensity matching approach: A tractable stochastic geometry approximation to systemlevel analysis of cellular networks," CoRR, vol. abs/1604.02683, 2016.

[26] M. Z. Win, P. C. Pinto, and L. A. Shepp, "A mathematical theory of network interference and its applications," Proc. IEEE, vol. 97, no. 2, pp. 205-230, Feb. 2009.

[27] P. C. Pinto and M. Z. Win, "Communication in a Poisson field of interferers-Part I: Interference distribution and error probability," IEEE Trans. Wireless Commun., vol. 9, no. 7, pp. 2176-2186, July 2010.

[28] L. H. Afify, H. ElSawy, T. Y. Al-Naffouri, and M. S. Alouini, "Unified tractable model for downlink MIMO cellular networks using stochastic geometry," in 2016 IEEE International Conference on Communications (ICC), May 2016, pp. 1-7.

[29] L. H. Afify, H. ElSawy, T. Y. Al-Naffouri, and M.-S. Alouini, "The influence of Gaussian signaling approximation on error performance in cellular networks," IEEE Commun. Lett., vol. 19, no. 12, pp. 22022205, Dec 2015.

[30] R. Vaze and R. W. Heath, "Transmission capacity of ad-hoc networks with multiple antennas using transmit stream adaptation and interference cancellation," IEEE Transactions on Information Theory, vol. 58, no. 2, pp. 780-792, Feb 2012.

[31] A. M. Hunter, J. G. Andrews, and S. Weber, "Transmission capacity of ad hoc networks with spatial diversity," December 2008, vol. 7, pp. 5058-5071.

[32] W. Choi, N. Himayat, S. Talwar, and M. Ho, "The effects of cochannel interference on spatial diversity techniques," in Wireless Communications and Networking Conference, 2007.WCNC 2007. IEEE, March 2007, pp. 1936-1941.

[33] H. ElSawy and E. Hossain, "On stochastic geometry modeling of cellular uplink transmission with truncated channel inversion power control," IEEE Trans. Wireless Commun., vol. 13, no. 8, pp. 44544469, Aug. 2014.

[34] L. Afify, H. ElSawy, T. Al-Naffouri, and M.-S. Alouini, "Error performance analysis in K-tier uplink cellular networks using a stochastic geometric approach," in Proc. of the IEEE ICC 4th International Workshop on Small Cell and $5 G$ Networks (SmallNets), London, UK, Jun. 2015.

[35] M. Haenggi, Stochastic Geometry for Wireless Networks, Cambridge University Press, 2012.

[36] H.S. Dhillon, R.K. Ganti, and J.G. Andrews, "Load-aware modeling and analysis of heterogeneous cellular networks," IEEE Trans. Wireless Commun., vol. 12, no. 4, pp. 1666-1677, Apr. 2013.

[37] H. ElSawy and E. Hossain, "On cognitive small cells in two-tier heterogeneous networks," in Proc. of the 9th International Workshop on Spatial Stochastic Models for Wireless Networks (SpaSWiN'2013), Tsukuba Science City, Japan, May 2013.

[38] M. K. Simon and M.-S. Alouini, Digital Communication over Fading Channels, vol. 95, Wiley-Interscience, 2005. 
[39] R.J. Marks, G.L. Wise, D.G. Haldeman, and J.L. Whited, "Detection in Laplace noise," IEEE Trans. on Aerosp. Electron. Syst., vol. AES14, no. 6, pp. 866-872, Nov 1978.

[40] S. Jiang and N.C. Beaulieu, "Precise ber computation for binary data detection in bandlimited white laplace noise," IEEE Trans. Commun., vol. 59, no. 6, pp. 1570-1579, June 2011.

[41] H. Soury, F. Yilmaz, and M.-S. Alouini, "Error rates of M-PAM and M-QAM in generalized fading and generalized gaussian noise environments," IEEE Commun. Lett., vol. 17, no. 10, pp. 1932-1935, October 2013.

[42] X. Cheng, C. X. Wang, H. Wang, X. Gao, X. H. You, D. Yuan, B. Ai, Q. Huo, L. Y. Song, and B. L. Jiao, "Cooperative MIMO channel modeling and multi-link spatial correlation properties," IEEE Journal on Selected Areas in Communications, vol. 30, no. 2, pp. 388-396, February 2012.

[43] M. Abramowitz and I. A. Stegun, Eds., Handbook of Mathematical Functions, Tenth Printing, Dover Publications, Dec. 1972.

[44] M. Di Renzo and P. Guan, "Stochastic geometry modeling of coverage and rate of cellular networks using the gil-pelaez inversion theorem," IEEE Communications Letters, vol. 18, no. 9, pp. 1575-1578, Sept 2014.

[45] H. Alzer, "On some inequalities for the incomplete gamma function," Mathematics of Computation, vol. 66, no. 218.

[46] K. Huang, R. W. Heath, and J. G. Andrews, "Space division multiple access with a sum feedback rate constraint," IEEE Transactions on Signal Processing, vol. 55, no. 7, pp. 3879-3891, July 2007.

[47] F. Olver, NIST handbook of mathematical functions, Cambridge University Press, 2010.

[48] Andrea Goldsmith, Wireless Communications, Cambridge University Press, New York, NY, USA, 2005.

[49] Y. M. Shobowale and K. A. Hamdi, "A unified model for interference analysis in unlicensed frequency bands," IEEE Trans. Wireless Commun., vol. 8, no. 8, pp. 4004-4013, Aug. 2009.

[50] K. A. Hamdi, "A useful lemma for capacity analysis of fading interference channels," IEEE Transactions on Communications, vol. 58, no. 2, pp. 411-416, 2010.

[51] B. Hassibi and B. M. Hochwald, "High-rate codes that are linear in space and time," IEEE Transactions on Information Theory, vol. 48, no. 7 , pp. 1804-1824, July 2002.

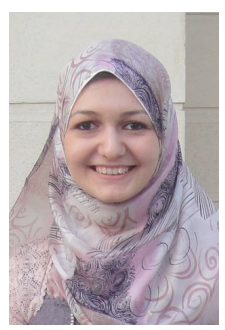

Laila Hesham Afify received her B.Sc. degree in Electrical Engineering from Cairo University, Egypt, in 2009. She worked as a research assistant at the Wireless Intelligent Network Center (WINC), Nile University, Giza, Egypt from 2009 to 2011. She received her M.Sc. degree in Wireless Communications from Nile University in 2011. Currently, she is pursuing her Ph.D. degree at King Abdullah University of Science and Technology (KAUST), Saudi Arabia. From 2011 to 2012, she was jointly affiliated with Nile University and the American University in Cairo as a junior scientist. She is also the recepient of the Best Paper Award in ICC 2015 workshop on Small Cells and 5G networks. Her research focuses on the use of stochastic geometry in cellular networks. Her main research interests include signal processing, stochastic geometry and cognitive radio systems.

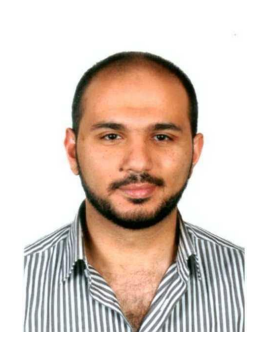

Hesham ElSawy (S'10, M'14) received the B.Sc. degree in Electrical Engineering from Assiut University, Assiut, Egypt, in 2006, the M.Sc. degree in Electrical Engineering from Arab Academy for Science and Technology, Cairo, Egypt, in 2009, and the Ph.D. degree in Electrical Engineering from the University of Manitoba, Winnipeg, MB Canada, in 2014. Currently, he is a postdoctoral fellow with the Computer, Electrical, and Mathematical Sciences and Engineering Division, King Abdullah University of Science and Technology (KAUST), Saudi Arabia, and an adjunct member at the school of Computer Science \& Engineering, York University, Canada. During the period of 2006-2010, he worked at the National Telecommunication Institute, Egypt, where he conducted professional training both at the national and international levels, as well as research on network planning. From 2010 to 2014, he worked with TRTech, Winnipeg, MB, Canada, as a Student Researcher. For his academic excellence, he has received several academic awards, including the NSERC Industrial Postgraduate Scholarship during the period of 2010-2013, and the TRTech Graduate Students Fellowship in the period of 2010-2014. He also received the best paper award in the ICC 2015 workshop on small cells and $5 \mathrm{G}$ networks. He is recognized as an exemplary reviewer by the IEEE Transactions of communication in 2015 \& 2016. His research interests include statistical modeling of wireless networks, stochastic geometry, and queueing analysis for wireless communication networks.

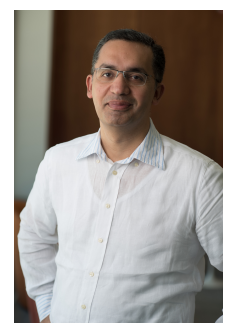

Tareq Al-Naffouri received the B.S. degrees in mathematics and electrical engineering (with first honors) from King Fahd University of Petroleum and Minerals, Dhahran, Saudi Arabia, the M.S. degree in electrical engineering from the Georgia Institute of Technology, Atlanta, in 1998, and the Ph.D. degree in electrical engineering from Stanford University, Stanford, CA, in 2004.

He was a visiting scholar at California Institute of Technology, Pasadena, CA, from January to August 2005 and during summer 2006. He was a Fulbright scholar at the University of Southern California from February to September 2008. He has held internship positions at NEC Research Labs, Tokyo, Japan, in 1998, Adaptive Systems Lab, University of California at Los Angeles in 1999, National Semiconductor, Santa Clara, CA, in 2001 and 2002, and Beceem Communications Santa Clara, CA, in 2004. He is currently an Associate at the Electrical Engineering Department, King Abdullah University of Science and Technology (KAUST). His research interests lie in the areas of sparse, adaptive, and statistical signal processing and their applications and in network information theory. He has over 160 publications in journal and conference proceedings, 9 standard contributions, 10 issued patents, and 6 pending.

Dr. Al-Naffouri is the recipient of the IEEE Education Society Chapter Achievement Award in 2008 and Al-Marai Award for innovative research in communication in 2009. Dr. Al-Naffouri has also been serving as an Associate Editor of Transactions on Signal Processing since August 2013.

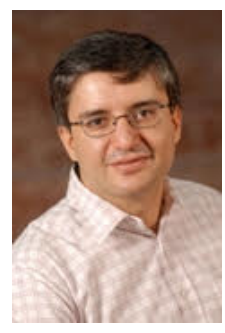

Mohamed-Slim Alouini (S'94, M'98, SM'03, F09) was born in Tunis, Tunisia. He received the $\mathrm{Ph} . \mathrm{D}$. degree in Electrical Engineering from the California Institute of Technology (Caltech), Pasadena, CA, USA, in 1998. He served as a faculty member in the University of Minnesota, Minneapolis, MN, USA, then in the Texas A\&M University at Qatar, Education City, Doha, Qatar before joining King Abdullah University of Science and Technology (KAUST), Thuwal, Makkah Province, Saudi Arabia as a Professor of Electrical Engineering in 2009. His current research interests include the modeling, design, and performance analysis of wireless communication systems. 